\title{
QUASILINEAR ELLIPTIC AND PARABOLIC ROBIN PROBLEMS ON LIPSCHITZ DOMAINS
}

\author{
ROBIN NITTKA
}

\begin{abstract}
We prove Hölder continuity up to the boundary for solutions of quasi-linear degenerate elliptic problems in divergence form, not necessarily of variational type, on Lipschitz domains with Neumann and Robin boundary conditions. This includes the $p$-Laplace operator for all $p \in(1, \infty)$, but also operators with unbounded coefficients. Based on the elliptic result we show that the corresponding parabolic problem is well-posed in the space $\mathrm{C}(\bar{\Omega})$ provided that the coefficients satisfy a mild monotonicity condition. More precisely, we show that the realization of the elliptic operator in $\mathrm{C}(\bar{\Omega})$ is m-accretive and densely defined. Thus it generates a non-linear strongly continuous contraction semigroup on $\mathrm{C}(\bar{\Omega})$.
\end{abstract}

\section{INTRODUCTION}

Given a bounded Lipschitz domain $\Omega$ in $\mathbb{R}^{N}$, we show that all weak solutions of certain degenerate quasi-linear elliptic problems are Hölder-continuous up to the boundary of $\Omega$, which generalizes the results in [25] to non-linear equations. More precisely, this is true for equations of the form

$$
\left\{\begin{aligned}
-\operatorname{div} A(x, u, \nabla u)+B(x, u, \nabla u)+\omega u=0 & & \text { on } \Omega \\
A(x, u, \nabla u) \cdot \nu+h(x, u)=0 & & \text { on } \partial \Omega
\end{aligned}\right.
$$

where $A: \Omega \times \mathbb{R} \times \mathbb{R}^{N} \rightarrow \mathbb{R}^{N}, B: \Omega \times \mathbb{R} \times \mathbb{R}^{N} \rightarrow \mathbb{R}$ and $h: \partial \Omega \times \mathbb{R} \rightarrow \mathbb{R}$ are measurable functions such that there exist constants $1<p<\infty$ and $0<\nu \leq \mu$ and non-negative functions $\psi_{1}, \psi_{2}$ and $\psi_{3}$ satisfying

$$
\begin{aligned}
z A(x, u, z) & \geq \nu|z|^{p}-\psi_{1}(x)|u|^{p}-\psi_{1}(x) \\
|A(x, u, z)| & \leq \mu|z|^{p-1}+\psi_{2}(x)|u|^{p-1}+\psi_{2}(x) \\
|B(x, u, z)| & \leq \psi_{3}(x)|z|^{p-1}+\psi_{1}(x)|u|^{p-1}+\psi_{1}(x) \\
|h(x, u)| & \leq \psi_{4}(x)|u|^{p-1}+\psi_{4}(x)
\end{aligned}
$$

for all $x \in \Omega, u \in \mathbb{R}$ and $z \in \mathbb{R}^{N}$, and such that

$$
\begin{array}{lllll}
\psi_{1} \in L^{\frac{N}{p-\varepsilon}}(\Omega), & \psi_{2} \in L^{\frac{N}{p-1}}(\Omega), & \psi_{3} \in L^{\frac{N}{1-\varepsilon}}(\Omega), & \psi_{4} \in L^{\frac{N-1}{p-1}}(\partial \Omega) & \text { if } p<N, \\
\psi_{1} \in L^{\frac{N}{N-\varepsilon}}(\Omega), & \psi_{2} \in L^{\frac{N}{N-1-\varepsilon}}(\Omega), & \psi_{3} \in L^{\frac{N}{1-\varepsilon}}(\Omega), & \psi_{4} \in L^{\frac{N-1}{N-1-\varepsilon}}(\partial \Omega) & \text { if } p=N, \\
\psi_{1} \in L^{1}(\Omega), & \psi_{2} \in L^{\frac{p}{p-1}}(\Omega), & \psi_{3} \in L^{p}(\Omega), \quad \psi_{4} \in L^{1}(\partial \Omega) & \text { if } p>N
\end{array}
$$

with some $\varepsilon \in(0,1)$. In particular, the $\Delta_{p}$-equation with Robin boundary conditions is included for all $p \in(1, \infty)$, together with a large variety of lower order perturbations, including unbounded coefficients. Also we do not require a variational structure of the equation. We refer to [20] and references therein for a short

Date: March 8, 2011.

2010 Mathematics Subject Classification. Primary: 35B65; Secondary: 35J25, 35K20.

Key words and phrases. Second order quasi-linear elliptic equations, Lipschitz domains, Robin boundary conditions, Hölder regularity, unbounded coefficients, parabolic equations, non-linear semigroup, space of continuous functions, Wentzell-Robin boundary conditions. 
account on applications for the $\Delta_{p}$-operator. The assumptions on the coefficients are optimal in that the regularity assumptions on $A$ and $B$ are the right ones within the class of $L^{p}$-functions for results about interior regularity 27], see also [23].

The other main result of this article is that under suitable conditions on the coefficients the unique solution of

$$
\left\{\begin{aligned}
u_{t}(t, x)-\operatorname{div} a(x, \nabla u(t, x))+b(x, u(t, x)) & =0 & & t>0, x \in \Omega \\
a(x, \nabla u(t, x)) \cdot \nu+h(x, u(x)) & =0 & & t>0, x \in \partial \Omega \\
u(0, x) & =u_{0}(x) & & x \in \Omega,
\end{aligned}\right.
$$

which we define in an $L^{2}$-sense, is continuous on the parabolic cylinder $[0, \infty) \times \bar{\Omega}$ whenever $u_{0} \in \mathrm{C}(\bar{\Omega})$. More precisely, we show that the corresponding elliptic operator is m-accretive on $\mathrm{C}(\bar{\Omega})$ and thus generates a non-linear contraction $\mathrm{C}_{0^{-}}$ semigroup on $\mathrm{C}(\bar{\Omega})$. In order to obtain this result we have to assume that $a, b$ and $h$ are Carathéodory functions that such $A(x, u, z):=a(x, z), B(x, u, z):=b(x, u)$ and $h$ satisfy the above conditions (1.2). Moreover, we assume the following mild monotonicity assumptions,

$$
\left\{\begin{aligned}
\left(z_{1}-z_{2}\right)\left(a\left(x, z_{1}\right)-a\left(x, z_{2}\right)\right) & \geq 0 \\
\left(u_{1}-u_{2}\right)\left(b\left(x, u_{1}\right)-b\left(x, u_{2}\right)\right) & \geq 0 \\
\left(u_{1}-u_{2}\right)\left(h\left(x, u_{1}\right)-h\left(x, u_{2}\right)\right) & \geq 0
\end{aligned}\right.
$$

which are much weaker than the standard monotonicity assumptions as considered for example in [24]. In particular, (1.4) includes the $\Delta_{p}$-operator for every $p \in$ $(1, \infty)$. Thus for all $p \in(1, \infty)$ the problem

$$
\left\{\begin{aligned}
u_{t}(t, x)-\Delta_{p} u(t, x)+b_{0}(x)|u|^{p-2} u & =0 & & t>0, x \in \Omega \\
|\nabla u(t, x)|^{p-2} \frac{\partial u(t, x)}{\partial \nu}+h_{0}(x)|u|^{p-2} u & =0 & & t>0, x \in \partial \Omega \\
u(0, x) & =u_{0}(x) & & x \in \Omega
\end{aligned}\right.
$$

is well-posed in $\mathrm{C}(\bar{\Omega})$ if $b_{0} \in L^{\infty}(\Omega)$ and $h_{0} \in L^{\infty}(\partial \Omega)$ are nonnegative. We also obtain a similar result for Wentzell-Robin boundary conditions. All of these results are based on the the author's PhD thesis [26].

There are several good reasons to study elliptic and parabolic equations in $\mathrm{C}(\bar{\Omega})$. On the one hand, for Dirichlet boundary conditions it is the natural space to formulate the boundary conditions, cf. [3- $[-5]$, and it is the natural space for maximum principles. But also for Neumann or Robin boundary conditions the space $\mathrm{C}(\bar{\Omega})$ is nicer in some respects than the $L^{q}$-spaces with $q \in[1, \infty)$. For instance, the composition operator $f \mapsto g \circ f$ is locally Lipschitz continuous in $\mathrm{C}(\bar{\Omega})$ whenever $g$ is locally Lipschitz continuous, but in general fails to map $L^{q}(\Omega)$ into $L^{q}(\Omega)$, so rapidly growing non-linear perturbations can more easily be handled in $\mathrm{C}(\bar{\Omega})$ than in $L^{q}(\Omega)$.

The results of this article are new regarding several aspects. Our results are valid for bounded Lipschitz domains, which form a strictly larger class than the strong Lipschitz domains, i.e., the domains that are locally the epigraph of a Lipschitz continuous functions. For example, the physically relevant example of the topologically regularized union of two crossing beams is a Lipschitz domain, but not a strong Lipschitz domain $\mid 17, \S 7.3]$. The class of Lipschitz domains has been studied a lot recently, see [12, 17, 18, 21], to name only a few articles.

Hölder continuity of solutions of linear equations with Robin boundary conditions on Lipschitz domains has been extensively studied, sometimes only in special cases, see for example [6, 10, 13, 25, 30]. The main elliptic result of this article, Theorem 4.4. seems to be new in the non-linear case even for smooth domains, but compare [16, 22] for corresponding results under more restrictive assumptions 
on the coefficients, which are obtained by different methods. The linear parabolic problem has been studied in 25, 30] in terms of semigroups. The non-linear case seems to be new, but see [14] for results under stronger regularity assumptions. Our result seems to be particularly interesting because we neither assume that the corresponding elliptic operator has a variational structure nor that it is strongly monotone.

The article is structured as follows. After introducing some notational conventions and basic properties of Lipschitz domains in Section 2 we show in Section 3 that every solution of (1.1) for $h=0$ is Hölder continuous, thus proving the main elliptic result for Neumann boundary conditions. The proof is based on a reflection argument that the author has used already for the linear case [25]. The general idea is much older, compare for example [29, Section 2.4.3], but has apparently not been exploited to this extent before.

In Section 4 we obtain a priori estimates for the Robin problem. We use Moser's iteration in a similar manner as in [8]. Our result is more general than those in [8] in that we allow general quasi-linear operators, but less general in that we restrict ourselves to Lipschitz domains. Combining these a priori estimates with our main result for problems with Neumann boundary conditions we extend the regularity result to general $h$.

Finally, in Section [5 me make use of the elliptic theory in order to show that the parabolic problem with Robin boundary conditions is well-posed in the space of continuous functions. The result is based on non-linear semigroup theory. Following the ideas in [2], we are able to apply our methods also to equations with WentzellRobin boundary conditions. We do not have to assume that the $L^{2}$-realization of the operator is a subdifferential, i.e., we do not assume that the corresponding elliptic problem has a variational formulation.

\section{NotATION AND PRELIMINARIES}

Throughout the article we follow the convention that constants denoted by $c$ are allowed to vary from one line to the next and may depend freely on the parameters $N, \Omega, p, \varepsilon$ and upper bounds for $\left\|\psi_{1}\right\|,\left\|\psi_{2}\right\|,\left\|\psi_{3}\right\|$ and $\left\|\psi_{4}\right\|$ in their respective spaces, as introduced in (1.2). Any additional dependencies are explicitly indicated by subscripts.

Definition 2.1. We say that an open set $\Omega \subset \mathbb{R}^{N}$ is a Lipschitz domain if for every $x \in \partial \Omega$ there exists an $\mathbb{R}^{N}$-neighborhood $V$ of $x$ and a bi-Lipschitz mapping $\psi$ from $V$ onto $(-1,1)^{N}$ such that $\psi(V \cap \Omega)=(-1,1)^{N-1} \times(0,1)$, i.e., $\psi$ is invertible and both $\psi$ and $\psi^{-1}$ are Lipschitz continuous. One says that $\bar{\Omega}$ is an $N$-dimensional, bounded Lipschitz submanifold of $\mathbb{R}^{N}$ with boundary.

Remark 2.2 ([15, §1.2.1]). Every domain with Lipschitz boundary is a Lipschitz domain, but the converse fails.

If $\Omega$ is a Lipschitz domain, then it is an extension domain [11, Theorem 7.25] which implies that $\mathrm{C}^{\infty}(\bar{\Omega})$ is dense in $W^{1, p}(\Omega)$ for $p \in[1, \infty)$ and that the usual Sobolev embeddings hold. The natural measure on the boundary of a Lipschitz domain is the $(N-1)$-dimensional Hausdorff measure. It is the unique measure for which the divergence theorem holds. This fact is easily transported from the reference domain $(-1,1)^{N-1} \times(0,1)$ to $\Omega$, see also [9, §5.8]. We agree that integrals over the boundary of a Lipschitz domain are always to be understood to be taken with respect to the $(N-1)$-dimensional Hausdorff measure, which we denote by $\sigma$ if the need arises.

Let $1 \leq q \leq \infty$. If we write $\|u\|_{L^{q}(\Omega)}$, where $u$ is a measurable function on $\Omega$, we regard that expression to equal infinity if $u \notin L^{q}(\Omega)$. For convenience we use the 
notation

$$
\|u\|_{L^{q}(\partial \Omega)}:=\left\|\left.u\right|_{\partial \Omega}\right\|_{L^{q}(\partial \Omega)}
$$

for functions $u \in W^{1, p}(\Omega)$, which admit a trace $\left.u\right|_{\partial \Omega} \in L^{p}(\partial \Omega)$, and again we define this expression to equal infinity if $\left.u\right|_{\partial \Omega} \notin L^{q}(\partial \Omega)$.

We will need a change of variables formula for boundary integrals. In order to prove it, we start with a few facts about the derivative of bi-Lipschitz mappings.

Lemma 2.3. Let $U \subset \mathbb{R}^{n}$ be open and let $\psi: U \rightarrow \mathbb{R}^{m}$ be a bi-Lipschitz mapping, $m \geq n$. Then $\psi$ is differentiable almost everywhere and the Jacobian $J \psi(x):=$ $\left(\operatorname{det}\left(\psi^{\prime}(x)^{T} \psi^{\prime}(x)\right)\right)^{1 / 2}$ of $\psi$ satisfies $\alpha \leq J \psi \leq \beta$ almost everywhere, where the constants $\alpha$ and $\beta$ depend only on $n, m$ and the Lipschitz constants of $\psi$ and $\psi^{-1}$. If $m=n$, then $\psi^{\prime}$ is invertible almost everywhere with uniformly bounded inverse and $\alpha \leq\left|\operatorname{det} \psi^{\prime}\right| \leq \beta$.

Proof. Rademacher's theorem asserts that $\psi$ is differentiable almost everywhere. More precisely, the entries of $\psi^{\prime}$ are essentially bounded by the Lipschitz constant of $\psi$, which proves the upper estimate for $J \psi$. As for the lower estimate, let $L>0$ be the Lipschitz constant of $\psi^{-1}$, so that $|\psi(y)-\psi(x)| \geq L^{-1}|x-y|$ for all $x, y \in U$. If $x$ is a point of differentiability of $\psi$, then

$$
L^{-1}|t v| \leq|\psi(x+t v)-\psi(x)|=\left|t \psi^{\prime}(x) v+o(t)\right| .
$$

For $t \rightarrow 0$ we obtain that $\left|\psi^{\prime}(x) v\right| \geq L^{-1}|v|$ almost everywhere for all $v \in \mathbb{R}^{n}$, hence

$$
|v|\left|\psi^{\prime}(x)^{T} \psi^{\prime}(x) v\right| \geq\left(\psi^{\prime}(x)^{T} \psi^{\prime}(x) v \mid v\right)=\left|\psi^{\prime}(x) v\right|^{2} \geq L^{-2}|v|^{2} .
$$

Thus the eigenvalues of $\psi^{\prime}(x)^{T} \psi^{\prime}(x)$ can be bounded from below by $L^{-2}$, which shows that $J \psi \geq L^{-n}$ holds almost everywhere.

Finally, if $m=n$, then the chain rule [31, Theorem 2.2.2] implies that

$$
\left(\psi^{-1}\right)^{\prime}(\psi(x))=\left(\psi^{\prime}(x)\right)^{-1} .
$$

Since the entries of $\left(\psi^{-1}\right)^{\prime}$ are essentially bounded, this proves that $\left(\psi^{\prime}\right)^{-1}$ is uniformly bounded outside a set of measure zero. The estimate for the determinant follows from $J \psi=\left|\operatorname{det} \psi^{\prime}\right|$.

We can now prove the following change of variables formula for boundary integrals.

Lemma 2.4. Let $\Omega_{1}$ and $\Omega_{2}$ be Lipschitz domains and $\psi: \Omega_{2} \rightarrow \Omega_{1}$ a bi-Lipschitz function. Then $\psi$ has a unique extension to $\bar{\Omega}_{1}$, and $\psi\left(\partial \Omega_{2}\right)=\partial \Omega_{1}$, where we identify $\psi$ with its extension. In this situation, there exists a measurable function $m: \Omega_{2} \rightarrow(0, \infty)$, which is unique up to nullsets, such that

$$
\int_{\partial \Omega_{1}} g=\int_{\partial \Omega_{2}}(g \circ \psi) m
$$

for all positive measurable functions $g$ on $\partial \Omega_{1}$ and hence for all integrable functions. Moreover, $0<\alpha \leq m \leq \beta$ almost everywhere with constants $\alpha$ and $\beta$ that depend only on $\psi, \Omega_{1}$ and $\Omega_{2}$.

Proof. The assertions about $\psi$ and the uniqueness of $m$ are clear. In order to show the existence of $m$, fix $y \in \partial \Omega_{2}$ and define $x:=\psi(y) \in \partial \Omega_{1}$. Fix neighborhoods $V_{2}$ of $y$ and $V_{1}$ of $x$ such that there exist bi-Lipschitz transformations $\psi_{1}: V_{1} \rightarrow(-1,1)^{N}$ and $\psi_{2}: V_{2} \rightarrow(-1,1)^{N}$ as in Definition 2.1. Without loss of generality we pick $V_{2}$ so small that $\psi\left(V_{2}\right) \subset V_{1}$. Write

$$
\varphi_{i}:=\left.\psi_{i}\right|_{\partial \Omega_{i} \cap V_{i}}: \partial \Omega_{i} \cap V_{i} \rightarrow H:=(-1,1)^{N-1} \times\{0\} .
$$


Then the bi-Lipschitz function $\varphi:=\varphi_{1} \circ \psi \circ \varphi_{2}^{-1}: H \rightarrow H$ is the local representation of $\left.\psi\right|_{\partial \Omega_{2}}$, and we regard $H$ as an open subset of $\mathbb{R}^{N-1}$. Then for every positive measurable function $f$ on $H$ we obtain that

$$
\int_{H} f=\int_{H}(f \circ \varphi)\left|\operatorname{det} \varphi^{\prime}\right|
$$

by the change of variables formula for Lipschitz functions [9, §3.3.3]. Also by the change of variables formula we obtain that

$$
\int_{H} f J \varphi_{i}^{-1}=\int_{\partial \Omega_{i}} f \circ \varphi_{i}
$$

for $i=1,2$, where $J \varphi_{i}^{-1}$ denotes the Jacobian of $\varphi_{i}^{-1}$ as a mapping from $H$ into $\mathbb{R}^{N}$. Combining these formulas, we see that

$$
\begin{aligned}
\int_{\partial \Omega_{1}} g & =\int_{H}\left(g \circ \varphi_{1}^{-1}\right) J \varphi_{1}^{-1}=\int_{H}\left(g \circ \varphi_{1}^{-1} \circ \varphi\right)\left(J \varphi_{1}^{-1} \circ \varphi\right)\left|\operatorname{det} \varphi^{\prime}\right| \\
& =\int_{\partial \Omega_{2}}\left(g \circ \varphi_{1}^{-1} \circ \varphi \circ \varphi_{2}\right) \frac{J \varphi_{1}^{-1} \circ \varphi \circ \varphi_{2}}{J \varphi_{2}^{-1} \circ \varphi_{2}}\left|\operatorname{det} \varphi^{\prime} \circ \varphi_{2}\right|
\end{aligned}
$$

for all positive measurable functions $g$ on $\partial \Omega_{1}$. By Lemma 2.3 , this implies the claim.

\section{Elliptic Neumann problems}

Let $\Omega \subset \mathbb{R}^{N}$ be a Lipschitz domain and $p \in(1, \infty)$. We prove that all weak solutions $u \in W^{1, p}(\Omega)$ of

$$
\left\{\begin{aligned}
-\operatorname{div} A(x, u, \nabla u)+B(x, u, \nabla u) & =f(x)-(\operatorname{div} F)(x) & & \text { on } \Omega \\
A(x, u, \nabla u) \cdot \nu & =g(x)+(F \cdot \nu)(x) & & \text { on } \partial \Omega
\end{aligned}\right.
$$

are Hölder continuous, provided $A$ satisfies the structure conditions (1.2) and $f, F$ and $g$ are in appropriate Lebesgue spaces, namely

$$
\left\{\begin{array}{llll}
f \in L^{\frac{N}{p-\varepsilon}}(\Omega), & F \in L^{\frac{N}{p-1}}\left(\Omega ; \mathbb{R}^{N}\right), & g \in L^{\frac{N-1}{p-1}}(\partial \Omega) & \text { if } p<N, \\
f \in L^{\frac{N}{N-\varepsilon}}(\Omega), & F \in L^{\frac{N}{N-1-\varepsilon}}\left(\Omega ; \mathbb{R}^{N}\right), & g \in L^{\frac{N-1}{N-1-\varepsilon}}(\partial \Omega) & \text { if } p=N, \\
f \in L^{1}(\Omega), & F \in L^{\frac{p}{p-1}}\left(\Omega ; \mathbb{R}^{N}\right), & g \in L^{1}(\partial \Omega) & \text { if } p>N .
\end{array}\right.
$$

We could absorb $f$ and $F$ (but not $g$ ) into the coefficients, but for the application we have in mind it turns out to be more convenient to write them down explicitly. By convention, $\|f\|,\|F\|$ and $\|g\|$ will always refer to the norms of $f, F$ and $g$ in the spaces indicated in (3.2).

Definition 3.1. We say that $u \in W^{1, p}(\Omega)$ is a weak solution of (3.1) if

$$
\int_{\Omega} \nabla \eta A(x, u, \nabla u)+\int_{\Omega} \eta B(x, u, \nabla u)=\int_{\Omega} \eta f+\int_{\Omega} \nabla \eta F+\int_{\partial \Omega} \eta g
$$

for all $\eta \in \mathrm{C}^{\infty}(\bar{\Omega})$. If (3.3) holds merely for all $\eta \in \mathrm{C}_{c}^{\infty}(\Omega)$, we say that $u$ is a solution of the equation given by the first line of (3.1), without any boundary conditions.

Remark 3.2. A function $u \in W^{1, p}(\Omega)$ is a weak solution of (3.1) if and only if (3.3) holds for all $\eta \in W^{1, p}(\Omega)$ since $\mathrm{C}^{\infty}(\bar{\Omega})$ is dense in $W^{1, p}(\Omega)$ and all expressions in (3.3) are continuous as $\eta$ varies in $W^{1, p}(\Omega)$, compare Proposition 5.1, where an even stronger assertion is proved. In what follows, we will use this fact frequently.

We deduce boundary regularity from the following interior regularity result, which is an immediate consequence of results due to Serrin. 
Theorem $3.3([27, \S 1.1, \S 1.4, \S 1.5])$. Let $A$ and $B$ satisfy the structure conditions (1.2) and let $f$ and $F$ be as in (3.2). Then there exists $\alpha \in(0,1)$ such that every weak solution $u \in W^{1, p}(\Omega)$ of

$$
-\operatorname{div} A(x, u, \nabla u)+B(x, u, \nabla u)=f(x)-(\operatorname{div} F)(x) \text { on } \Omega
$$

is in $\mathrm{C}_{\text {loc }}^{0, \alpha}(\Omega)$. Moreover, for every relatively compact subdomain $\omega \subset \Omega$ there exists $c_{\alpha, \omega} \geq 0$ such that

$$
\|u\|_{C^{0, \alpha}(\omega)} \leq c_{\alpha, \omega}\left(\|f\|^{\frac{1}{p-1}}+\|F\|^{\frac{1}{p-1}}+\|u\|_{L^{p}(\Omega)}\right)+c_{\alpha, \omega}
$$

holds for all weak solutions $u \in W^{1, p}(\Omega)$ of (3.4).

In order to apply Theorem 3.3 we extend the solutions of 3.1 locally along the boundary of $\Omega$ and show that the extension satisfies an elliptic equation on the larger domain. Then interior regularity of the extended function implies boundary regularity of the original function.

It is convenient to carry over the function to the reference domain $(-1,1)^{N-1} \times$ $(0,1)$ and to extend the resulting function on that domain. As a first step, we show that the structural properties of the equation are preserved under bi-Lipschitz transformations.

Proposition 3.4. Let $\Omega_{1}$ and $\Omega_{2}$ be Lipschitz domains in $\mathbb{R}^{N}$ and let $\psi: \Omega_{2} \rightarrow \Omega_{1}$ be a bi-Lipschitz bijection. Let $u \in W^{1, p}\left(\Omega_{1}\right)$ and $v:=u \circ \psi$. Given functions $A$, $B, f, F$ and $g$ as in (1.2) and (3.2), define

$$
\begin{aligned}
& \hat{A}(x, u, z):=\left(\psi^{\prime}(x)\right)^{-1} A\left(\psi(x), u, z \psi^{\prime}(x)^{-1}\right)\left|\operatorname{det} \psi^{\prime}(x)\right| \\
& \hat{B}(x, u, z):=B\left(\psi(x), u, z \psi^{\prime}(x)^{-1}\right)\left|\operatorname{det} \psi^{\prime}(x)\right|
\end{aligned}
$$

for $x \in \Omega_{2}, u \in \mathbb{R}$ and row vectors $z \in \mathbb{R}^{N}$. Moreover, let $\hat{f}:=(f \circ \psi)\left|\operatorname{det} \psi^{\prime}\right|$, $\hat{F}:=\left(\psi^{\prime}\right)^{-1}(F \circ \psi)\left|\operatorname{det} \psi^{\prime}\right|$ and $\hat{g}:=(g \circ \psi) m$ with $m$ as in Lemma 2.4.

(a) The function $v$ is in $W^{1, p}\left(\Omega_{2}\right)$ with $\nabla v=(\nabla u \circ \psi) \psi^{\prime}$ almost everywhere, and the functions $\hat{f}, \hat{F}$ and $\hat{g}$ are in Lebesgue spaces with the same exponent as $f, F$ and $g$, respectively. More precisely, $\|\hat{f}\| \leq c_{\psi}\|f\|,\|\hat{F}\| \leq c_{\psi}\|F\|$ and $\|\hat{g}\| \leq c_{\psi}\|g\|$.

(b) The functions $\hat{A}$ and $\hat{B}$ satisfy the structure conditions (1.2) on $\Omega_{2}$, where the parameters depend only on $\psi$ and the parameters for $A$ and $B$.

(c) If $u \in W^{1, p}\left(\Omega_{1}\right)$ satisfies

$$
\int_{\Omega_{1}} \nabla \eta A(x, u, \nabla u)+\int_{\Omega_{1}} \eta B(x, u, \nabla u)=\int_{\Omega_{1}} \eta f+\int_{\Omega_{1}} \nabla \eta F+\int_{\partial \Omega_{1}} \eta g
$$

for all $\eta \in \mathrm{C}_{c}^{\infty}\left(\Omega_{1} \cup \Gamma\right)$ with some relatively open set $\Gamma \subset \partial \Omega_{1}$ then $v \in W^{1, p}\left(\Omega_{2}\right)$ satisfies

$$
\int_{\Omega_{2}} \nabla \tilde{\eta} \hat{A}(x, v, \nabla v)+\int_{\Omega_{2}} \tilde{\eta} \hat{B}(x, v, \nabla v)=\int_{\Omega_{2}} \tilde{\eta} \hat{f}+\int_{\Omega_{2}} \nabla \tilde{\eta} \hat{F}+\int_{\partial \Omega_{2}} \tilde{\eta} \hat{g}
$$

for all $\tilde{\eta} \in \mathrm{C}_{c}^{\infty}\left(\Omega_{2} \cup \psi^{-1}(\Gamma)\right)$.

Proof. The assertions in (国) follow from the chain rule for Sobolev functions 31, Theorem 2.2.2], the change of coordinates formula for Lipschitz transformations [9, $\S 3.3 .3]$ and Lemma 2.4. We have also used that $\left|\operatorname{det} \psi^{\prime}\right|$ is bounded from below and above, see Lemma 2.3 .

In order to check (b) we fix $u \in \mathbb{R}$ and a row vector $z \in \mathbb{R}^{N}$. Then

$$
\begin{aligned}
z \hat{A}(x, u, z) & =z\left(\psi^{\prime}(x)\right)^{-1} A\left(\psi(x), u, z\left(\psi^{\prime}(x)\right)^{-1}\right)\left|\operatorname{det} \psi^{\prime}(x)\right| \\
& \geq\left(\nu\left|z \psi^{\prime}(x)^{-1}\right|^{p}-\psi_{1}(\psi(x))|u|^{p}-\psi_{1}(\psi(x))\right)\left|\operatorname{det} \psi^{\prime}(x)\right|
\end{aligned}
$$




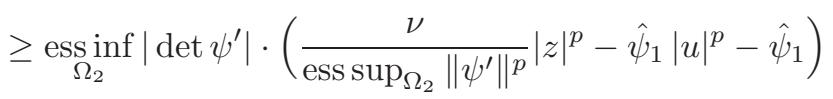

with the function $\hat{\psi}_{1}:=\psi_{1} \circ \psi$ possessing the same degree of integrability as $\psi_{1}$. Similarly,

$$
\begin{aligned}
& |\hat{A}(x, u, z)| \leq \underset{\Omega_{2}}{\operatorname{ess} \sup }\left|\operatorname{det} \psi^{\prime}\right| s\left(\mu s^{p-1}|z|^{p-1}+\hat{\psi}_{2}|u|^{p-1}+\hat{\psi}_{2}\right) \\
& |\hat{B}(x, u, z)| \leq \underset{\Omega_{2}}{\operatorname{ess} \sup }\left|\operatorname{det} \psi^{\prime}\right|\left(s^{p-1} \hat{\psi}_{3}|z|^{p-1}+\hat{\psi}_{1}|u|^{p-1}+\hat{\psi}_{1}\right)
\end{aligned}
$$

where $s:=\operatorname{ess}_{\Omega_{2}}\left\|\left(\psi^{\prime}\right)^{-1}\right\|, \hat{\psi}_{2}:=\psi_{2} \circ \psi$ and $\hat{\psi}_{3}:=\psi_{3} \circ \psi$. Hence $\hat{A}$ and $\hat{B}$ satisfy (1.2) on $\Omega_{2}$.

As for (ㄷ), let $\Gamma \subset \partial \Omega_{1}$ be relatively open and assume that $u$ satisfies (3.6) for all $\eta \in \mathrm{C}_{c}^{\infty}\left(\Omega_{1} \cup \Gamma\right)$. Then by denseness (3.6) is fulfilled for all $\eta \in W_{0}^{1, p}\left(\Omega_{1} \cup \Gamma\right)$, the closure of $\mathrm{C}_{c}^{\infty}\left(\Omega_{1} \cup \Gamma\right)$ in $W^{1, p}(\Omega)$. Let $\tilde{\eta}$ be in $\mathrm{C}_{c}^{\infty}\left(\Omega_{2} \cup \psi^{-1}(\Gamma)\right)$ and write $\eta:=$ $\tilde{\eta} \circ \psi^{-1}$. Then by a standard smoothing argument we obtain that $\eta \in W_{0}^{1, p}\left(\Omega_{1} \cup \Gamma\right)$. Moreover,

$$
\nabla \eta=\left(\nabla \tilde{\eta} \circ \psi^{-1}\right)\left(\psi^{-1}\right)^{\prime}=\left(\nabla \tilde{\eta} \circ \psi^{-1}\right)\left(\psi^{\prime} \circ \psi^{-1}\right)^{-1}
$$

by the chain rule. Hence

$$
\begin{aligned}
\int_{\Omega_{2}} \nabla & \tilde{\eta}(x) \hat{A}(x, v(x), \nabla v(x))+\int_{\Omega_{2}} \tilde{\eta}(x) \hat{B}(x, v(x), \nabla v(x)) \\
= & \int_{\Omega_{2}} \nabla \eta(\psi(x)) A(\psi(x), u(\psi(x)), \nabla u(\psi(x)))\left|\operatorname{det} \psi^{\prime}(x)\right| \\
& \quad+\int_{\Omega_{2}} \eta(\psi(x)) B(\psi(x), u(\psi(x)), \nabla u(\psi(x)))\left|\operatorname{det} \psi^{\prime}(x)\right| \\
= & \int_{\Omega_{1}} \nabla \eta(x) A(x, u(x), \nabla u(x))+\int_{\Omega_{1}} \eta(x) B(x, u(x), \nabla u(x)) \\
= & \int_{\Omega_{1}} \eta f+\int_{\Omega_{1}} \nabla \eta F+\int_{\partial \Omega_{1}} \eta g \\
= & \int_{\Omega_{2}} \tilde{\eta}(f \circ \psi)\left|\operatorname{det} \psi^{\prime}\right|+\int_{\Omega_{2}} \nabla \tilde{\eta}\left(\psi^{\prime}\right)^{-1}(F \circ \psi)\left|\operatorname{det} \psi^{\prime}\right|+\int_{\partial \Omega_{2}} \tilde{\eta}(g \circ \psi) m \\
= & \int_{\Omega_{2}} \tilde{\eta} \hat{f}+\int_{\Omega_{2}} \nabla \tilde{\eta} \hat{F}+\int_{\partial \Omega_{2}} \tilde{\eta} \hat{g},
\end{aligned}
$$

where by the change of variables formula for integrals over the domain as well as over its boundary, see Lemma 2.4.

Remark 3.5. In part ( $\mathrm{C}$ ) of Proposition 3.4 the values of $g$ on $\partial \Omega_{1} \backslash \Gamma$ do not appear in the assertions. Hence we can apply the result also if $g$ is given only on $\Gamma$ and is left undefined on the remaining part of $\partial \Omega_{1}$ by artificially defining $g:=0$ on $\partial \Omega_{1} \backslash \Gamma$.

We will also use the following representation of boundary integrals as integrals over the domain itself.

Lemma 3.6. Let $\Omega^{\prime}:=(-1,1)^{N}$ and $H:=(-1,1)^{N} \times\{0\}$, and let $g \in L^{q}(H)$, $q \in[1, \infty)$. There exist functions $k$ and $K$ in the spaces

$$
\left\{\begin{array}{lll}
k \in L^{\frac{q N}{N-1}}\left(\Omega^{\prime}\right), & K \in L^{\frac{q N}{N-1}}\left(\Omega^{\prime} ; \mathbb{R}^{N}\right) & \text { if } q>1 \\
k \in L^{\frac{N}{N-1+\varepsilon}}\left(\Omega^{\prime}\right), & K \in L^{\frac{N}{N-1+\varepsilon}}\left(\Omega^{\prime} ; \mathbb{R}^{N}\right) & \text { if } q=1
\end{array}\right.
$$

where $\varepsilon \in(0,1)$ is arbitrary, such that $k$ and $K$ satisfy

$$
\int_{H} \eta g=\int_{\Omega^{\prime}} \eta k+\int_{\Omega^{\prime}} \nabla \eta K
$$


for all $\eta \in \mathrm{C}_{c}^{\infty}\left(\Omega^{\prime}\right)$. Moreover, $\|k\|+\|K\| \leq c_{q, \varepsilon}\|g\|_{L^{q}(H)}$.

Proof. Define the linear functional $\varphi: \mathrm{C}_{c}^{\infty}\left(\Omega^{\prime}\right) \rightarrow \mathbb{R}$ by $\varphi(\eta):=\int_{H} \eta g$. If $q>1$, then

$$
|\varphi(\eta)| \leq\|\eta\|_{L^{\frac{q}{q-1}(H)}}\|g\|_{L^{q}(H)} \leq c_{q}\|\eta\|_{W^{1, \frac{q N}{q N-N+1}\left(\Omega^{\prime}\right)}}\|g\|_{L^{q}(H)}
$$

by the Sobolev embedding theorems. Similarly, if $q=1$, then

$$
|\varphi(\eta)| \leq\|\eta\|_{L^{\infty}(H)}\|g\|_{L^{1}(H)} \leq c_{\varepsilon}\|\eta\|_{W^{1, \frac{N}{1-\varepsilon}}\left(\Omega^{\prime}\right)}\|g\|_{L^{1}(H)}
$$

for every $\varepsilon>0$. Hence $\varphi$ extends to a continuous linear functional on $W_{0}^{1, \frac{q N}{q N-N+1}}\left(\Omega^{\prime}\right)$ or $W_{0}^{1, \frac{N}{1-\varepsilon}}\left(\Omega^{\prime}\right)$, respectively, which implies the claim, see [31, §4.3].

We now prove the main result of this section: every weak solution of (3.1) is Hölder continuous up to the boundary of $\Omega$.

Theorem 3.7. Let $\Omega$ be a Lipschitz domain and assume (1.2) and (3.2). Then there exist $\alpha \in(0,1)$ and $c_{\alpha} \geq 0$ such that every weak solution $u \in W^{1, p}(\Omega)$ of (3.1) is in $\mathrm{C}^{0, \alpha}(\Omega)$ and satisfies

$$
\|u\|_{C^{0, \alpha}(\Omega)} \leq c_{\alpha}\left(\|f\|^{\frac{1}{p-1}}+\|F\|^{\frac{1}{p-1}}+\|g\|^{\frac{1}{p-1}}+\|u\|_{L^{p}(\Omega)}\right)+c_{\alpha} .
$$

Proof. Let $u \in W^{1, p}(\Omega)$ be a weak solution of (3.1). Let $z$ be in $\partial \Omega$ and fix $V$ and $\psi: V \rightarrow(-1,1)^{N}$ as in Definition 2.1. We will show that $u$ is Hölder continuous in a neighborhood $V_{z} \subset V$ of $z$. Since $u$ solves (3.1) we know in particular that

$$
\int_{\Omega} \nabla \eta A(x, u, \nabla u)+\int_{\Omega} \eta B(x, u, \nabla u)=\int_{\Omega} \eta f+\int_{\Omega} \nabla \eta F+\int_{\partial \Omega} \eta g
$$

for all $\eta \in \mathrm{C}_{c}^{\infty}(\Omega \cup(V \cap \partial \Omega))$. Write $\Omega_{1}:=\psi(\Omega \cap V)=(-1,1)^{N-1} \times(0,1)$. Then by Proposition 3.4, see also Remark 3.5, the function $v_{1}:=u \circ \psi^{-1}$ satisfies

$$
\int_{\Omega_{1}} \nabla \eta \hat{A}_{1}\left(x, v_{1}, \nabla v_{1}\right)+\int_{\Omega_{1}} \eta \hat{B}_{1}\left(x, v_{1}, \nabla v_{1}\right)=\int_{\Omega_{1}} \eta \hat{f}_{1}+\int_{\Omega_{1}} \nabla \eta \hat{F}_{1}+\int_{\partial \Omega_{1}} \eta \hat{g}_{1}
$$

for all $\eta \in \mathrm{C}_{c}^{\infty}\left((-1,1)^{N-1} \times[0,1)\right)$ with functions $\hat{A}_{1}, \hat{B}_{1}, \hat{f}_{1}, \hat{F}_{1}$ and $\hat{g}_{1}$ that satisfy the conditions (1.2) and (3.2),

Define the reflection $\psi_{0}: \mathbb{R}^{N} \rightarrow \mathbb{R}^{N}$ by

$$
\psi_{0}\left(x_{1}, \ldots, x_{N-1}, x_{N}\right):=\left(x_{1}, \ldots, x_{N-1},-x_{N}\right) .
$$

Then $v_{2}:=v_{1} \circ \psi_{0} \in W^{1, p}\left(\Omega_{2}\right)$ satisfies

$$
\int_{\Omega_{2}} \nabla \eta \hat{A}_{2}\left(x, v_{2}, \nabla v_{2}\right)+\int_{\Omega_{2}} \eta \hat{B}_{2}\left(x, v_{2}, \nabla v_{2}\right)=\int_{\Omega_{2}} \eta \hat{f}_{2}+\int_{\Omega_{2}} \nabla \eta \hat{F}_{2}+\int_{\partial \Omega_{2}} \eta \hat{g}_{2}
$$

for all $\eta \in \mathrm{C}_{c}^{\infty}\left((-1,1)^{N-1} \times(-1,0]\right)$ by Proposition 3.4 with functions $\hat{A}_{2}, \hat{B}_{2}, \hat{f}_{2}$, $\hat{F}_{2}$ and $\hat{g}_{2}$ that satisfy the conditions (1.2) and (3.2).

Define $\Omega_{0}:=(-1,1)^{N}$ and regard

$$
\begin{array}{ll}
v:=v_{1} \mathbb{1}_{\Omega_{1}}+v_{2} \mathbb{1}_{\Omega_{2}}, & \\
\hat{A}:=\hat{A}_{1} \mathbb{1}_{\Omega_{1}}+\hat{A}_{2} \mathbb{1}_{\Omega_{2}}, & \hat{B}:=\hat{B}_{1} \mathbb{1}_{\Omega_{1}}+\hat{B}_{2} \mathbb{1}_{\Omega_{2}}, \\
\hat{f}:=\hat{f}_{1} \mathbb{1}_{\Omega_{1}}+\hat{f}_{2} \mathbb{1}_{\Omega_{2}}, & \hat{F}:=\hat{F}_{1} \mathbb{1}_{\Omega_{1}}+\hat{F}_{2} \mathbb{1}_{\Omega_{2}}
\end{array}
$$

as functions on $\Omega_{0}$. Then $\hat{A}, \hat{B}, \hat{f}, \hat{F}$ and $\hat{g}$ satisfy the conditions (1.2) and (3.2). Moreover, $v \in W^{1, p}\left(\Omega_{0}\right)$ and $\nabla v=\nabla v_{1} \mathbb{1}_{\Omega_{1}}+\nabla v_{2} \mathbb{1}_{\Omega_{2}}$. In fact, the Gauss-Green 
theorem [9, §5.8] shows that for all $\eta \in \mathrm{C}_{c}^{\infty}\left(\Omega_{0}\right)$ we have

$$
\begin{aligned}
\int_{\Omega_{0}} \nabla \eta v & =\int_{\Omega_{1}} \nabla \eta v_{1}+\int_{\Omega_{2}} \nabla \eta v_{2} \\
& =\int_{\partial \Omega_{1}} \eta v_{1} \nu_{\Omega_{1}}-\int_{\Omega_{1}} \eta \nabla v_{1}+\int_{\partial \Omega_{2}} \eta v_{2} \nu_{\Omega_{2}}-\int_{\Omega_{2}} \eta \nabla v_{2} \\
& =-\int_{\Omega_{1}} \eta \nabla v_{1}-\int_{\Omega_{2}} \eta \nabla v_{2}
\end{aligned}
$$

since $v_{1}=v_{2}$ on the intersection of the boundaries and the outer normals equal $\nu_{\Omega_{1}}=-e_{N}$ and $\nu_{\Omega_{2}}=e_{N}$ on that set, where $e_{N}$ denotes the $N^{\text {th }}$ unit vector in $\mathbb{R}^{N}$. In addition, $\eta$ vanishes on the remaining parts of $\partial \Omega_{1}$ and $\partial \Omega_{2}$ by assumption.

Using in addition Lemma 3.6 we thus obtain that

$$
\begin{aligned}
\int_{\Omega_{0}} & \nabla \eta \hat{A}(x, v, \nabla v)+\int_{\Omega_{0}} \eta \hat{B}(x, v, \nabla v) \\
\quad= & \int_{\Omega_{1}} \eta \hat{f}_{1}+\int_{\Omega_{1}} \nabla \eta \hat{F}_{1}+\int_{\partial \Omega_{1}} \eta \hat{g}_{1}+\int_{\Omega_{2}} \eta \hat{f}_{2}+\int_{\Omega_{2}} \nabla \eta \hat{F}_{2}+\int_{\partial \Omega_{2}} \eta \hat{g}_{2} \\
= & \int_{\Omega_{0}} \eta(\hat{f}+k)+\int_{\Omega_{0}} \nabla \eta(\hat{F}+K),
\end{aligned}
$$

for all $\eta \in \mathrm{C}_{c}^{\infty}\left(\Omega_{0}\right)$ with functions $k \in L^{q}\left(\Omega_{0}\right)$ and $K \in L^{q}\left(\Omega_{0} ; \mathbb{R}^{N}\right)$, where $q:=\frac{N}{p-1}$ for $p<N, q:=\frac{N}{N-1-\varepsilon}$ for $p=N$ and $q:=\frac{p}{p-1}$ for $p>N$. Thus $v \in W^{1, p}\left(\Omega_{0}\right)$ is a weak solution of

$$
-\operatorname{div} \hat{A}(x, v, \nabla v)+\hat{B}(x, v, \nabla v)=(\hat{f}+k)-\operatorname{div}(\hat{F}+K) \text { on } \Omega_{0},
$$

where the coefficients $\hat{A}$ and $\hat{B}$ and the right hand side $\hat{f}+k$ and $\hat{F}+K$ satisfy the assumptions of Theorem 3.3. Consequently, there exists $\alpha \in(0,1)$ such that

$$
\begin{aligned}
\left\|v_{1}\right\|_{\mathrm{C}^{0, \alpha}\left(\left(-\frac{1}{2}, \frac{1}{2}\right)^{N-1} \times\left(0, \frac{1}{2}\right)\right)} & \leq\|v\|_{\mathrm{C}^{0, \alpha}\left(\left(-\frac{1}{2}, \frac{1}{2}\right)^{N}\right)} \\
& \leq c_{\alpha}\left(\|\hat{f}+k\|^{\frac{1}{p-1}}+\|F+K\|^{\frac{1}{p-1}}+\|v\|_{L^{p}\left(\Omega_{0}\right)}\right)+c_{\alpha} \\
& \leq c_{\alpha}\left(\|f\|^{\frac{1}{p-1}}+\|F\|^{\frac{1}{p-1}}+\|g\|^{\frac{1}{p-1}}+\|u\|_{L^{p}(\Omega)}\right)+c_{\alpha},
\end{aligned}
$$

see also part (a) of Proposition 3.4 Since $u=v_{1} \circ \psi$ and $\psi$ is Lipschitz continuous on $V$, we have shown that there exists a neighborhood $V_{z} \subset V$ of $z$ such that $\left.u\right|_{V_{z}} \in \mathrm{C}^{0, \alpha}\left(V_{z}\right)$ and

$$
\|u\|_{\mathrm{C}^{0, \alpha}\left(V_{z}\right)} \leq c_{\alpha}\left(\|f\|^{\frac{1}{p-1}}+\|F\|^{\frac{1}{p-1}}+\|g\|^{\frac{1}{p-1}}+\|u\|_{L^{p}(\Omega)}\right)+c_{\alpha} .
$$

Since $\partial \Omega$ is compact, there exist finitely many $z_{i} \in \partial \Omega$ such that $\partial \Omega \subset \bigcup_{i=1}^{m} V_{z_{i}}$. Set $\omega_{i}:=V_{z_{i}} \cap \Omega$ and $\omega_{0}:=\Omega \backslash \bigcup_{i=1}^{m} \omega_{i} \Subset \Omega$. Then $\left.u\right|_{\omega_{0}} \in \mathrm{C}^{0, \alpha_{0}}\left(\omega_{0}\right)$ by Theorem 3.3 and $\left.u\right|_{\omega_{i}} \in \mathrm{C}^{0, \alpha_{i}}\left(\omega_{i}\right)$ for $i=1, \ldots, m$ by what we have just shown. Thus we have proved that $u \in \mathrm{C}^{0, \alpha}(\Omega)$ for $\alpha:=\min _{i=1, \ldots, N} \alpha_{i}$, and more precisely we have shown that 3.8 holds.

\section{Elliptic Robin PRoblems}

Let $\Omega \subset \mathbb{R}^{N}$ be a Lipschitz domain. In this section we prove that all weak solutions $u \in W^{1, p}(\Omega)$ of

$$
\left\{\begin{aligned}
-\operatorname{div} A(x, u, \nabla u)+B(x, u, \nabla u)+\omega u & =f(x)-(\operatorname{div} F)(x) & & \text { on } \Omega \\
A(x, u, \nabla u) \cdot \nu+h(x, u) & =g(x)+(F \cdot \nu)(x) & & \text { on } \partial \Omega
\end{aligned}\right.
$$

are Hölder continuous, provided $A, B$ and $h$ satisfy the structure conditions (1.2), $f, F$ and $g$ are as in (3.2) and $\omega$ is a nonnegative constant. Like for Neumann 
boundary conditions, we say that $u \in W^{1, p}(\Omega)$ is a weak solution of (4.1) if

$\int_{\Omega} \nabla \eta A(x, u, \nabla u)+\int_{\Omega} \eta B(x, u, \nabla u)+\int_{\partial \Omega} \eta h(x, u)=\int_{\Omega} \eta f+\int_{\Omega} \nabla \eta F+\int_{\partial \Omega} \eta g$

holds for all $\eta \in \mathrm{C}^{\infty}(\bar{\Omega})$ or, equivalently, for all $\eta \in W^{1, p}(\Omega) \cap L^{2}(\Omega)$.

We start by proving $L^{q}$-bounds for solutions of the slightly simpler equation

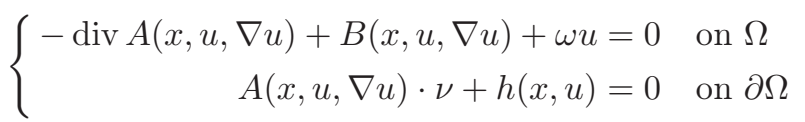

via Moser's iteration technique. If we assume some strong monotonicity of the coefficients, the a priori estimates could be obtained via an elegant interpolation argument, see [26]. But in order to cover the general case we have to use the iteration procedure instead.

We need the following lemma, whose easy proof we omit. But compare 28, Proposition II.5.2] for a similar argument.

Lemma 4.1. Let $X, Y$ and $Z$ be Banach spaces. Assume that $X$ is reflexive. Let $T: X \rightarrow Y$ be a compact linear operator and let $S: X \rightarrow Z$ be an injective bounded linear operator. Then for every $\delta>0$ there exists $c_{\delta}>0$ such that

$$
\|T x\|_{Y} \leq \delta\|x\|_{X}+c_{\delta}\|S x\|_{Z}
$$

holds for all $x \in X$.

Proposition 4.2. If $u \in W^{1, p}(\Omega)$ solves (4.2), then

$$
\|u\|_{W^{1, p}(\Omega)} \leq c\|u\|_{L^{p}(\Omega)}+c
$$

and

for every $q \in[p, \infty)$.

$$
\|u\|_{L^{q}(\Omega)}+\|u\|_{L^{q}(\partial \Omega)} \leq c_{q}\|u\|_{L^{p}(\Omega)}+c_{q}
$$

Proof. Let $u \in W^{1, p}(\Omega)$ be a weak solution of (4.2), and let $q \in[p, \infty)$ be arbitrary. Fix $\alpha \geq 1$ and define

$v_{\alpha}:=\left((|u|+1)^{q-p+1}-1\right) \operatorname{sgn}(u) \mathbb{1}_{\{|u| \leq \alpha\}}+\frac{(\alpha+1)^{q-p+1}-1}{\alpha+1}(|u|+1) \operatorname{sgn}(u) \mathbb{1}_{\{|u|>\alpha\}}$

and

$$
w_{\alpha}:=(|u|+1)^{\frac{q}{p}} \mathbb{1}_{\{|u| \leq \alpha\}}+(\alpha+1)^{\frac{q-p}{p}}(|u|+1) \mathbb{1}_{\{|u|>\alpha\}} .
$$

Then by the chain rule [31, Theorem 2.1.11] the functions $v_{\alpha}$ and $w_{\alpha}$ are in $W^{1, p}(\Omega)$ with weak derivatives

$$
\nabla v_{\alpha}=(q-p+1)(|u|+1)^{q-p} \nabla u \mathbb{1}_{\{|u| \leq \alpha\}}+\frac{(\alpha+1)^{q-p+1}-1}{\alpha+1} \nabla u \mathbb{1}_{\{|u|>\alpha\}}
$$

and

$$
\left|\nabla w_{\alpha}\right|=\frac{q}{p}(|u|+1)^{\frac{q-p}{p}}|\nabla u| \mathbb{1}_{\{|u| \leq \alpha\}}+(\alpha+1)^{\frac{q-p}{p}}|\nabla u| \mathbb{1}_{\{|u|>\alpha\}},
$$

hence

$$
\left(\frac{p}{q}\right)^{p}\left|\nabla w_{\alpha}\right|^{p} \leq(|u|+1)^{q-p}|\nabla u|^{p} \mathbb{1}_{\{|u| \leq \alpha\}}+(\alpha+1)^{q-p}|\nabla u|^{p} \mathbb{1}_{\{|u|>\alpha\}}
$$

We will also need that

$$
\frac{1}{2}(\alpha+1)^{q-p} \leq \frac{(\alpha+1)^{q-p+1}-1}{\alpha+1} \leq(\alpha+1)^{q-p},
$$

which follows from the fact that $(\alpha+1)^{q-p+1} \geq 2$. 
From (4.5), (4.6), (4.7) and (1.2) we obtain that

$$
\begin{aligned}
\int_{\Omega} & \nabla v_{\alpha} A(x, u, \nabla u) \\
\geq & (q-p+1) \int_{\{|u| \leq \alpha\}}(|u|+1)^{q-p}\left(\nu|\nabla u|^{p}-\psi|u|^{p}-\psi_{1}\right) \\
& +\frac{(\alpha+1)^{q-p+1}-1}{\alpha+1} \int_{\{|u|>\alpha\}}\left(\nu|\nabla u|^{p}-\psi_{1}|u|^{p}-\psi_{1}\right) \\
\geq & \nu \int_{\{|u| \leq \alpha\}}(|u|+1)^{q-p}|\nabla u|^{p}-2(q-p+1) \int_{\{|u| \leq \alpha\}} \psi_{1}(|u|+1)^{q} \\
& +\frac{\nu}{2} \int_{\{|u|>\alpha\}}(\alpha+1)^{q-p}|\nabla u|^{p}-2(q-p+1) \int_{\{|u|>\alpha\}} \psi_{1}(\alpha+1)^{q-p}(|u|+1)^{p} \\
\geq & \frac{\nu}{2}\left(\frac{p}{q}\right)^{p} \int_{\Omega}\left|\nabla w_{\alpha}\right|^{p}-2(q-p+1) \int_{\Omega} \psi_{1} w_{\alpha}^{p} .
\end{aligned}
$$

Similarly, we see that

$$
\begin{aligned}
& \left|\int_{\Omega} v_{\alpha} B(x, u, \nabla u)\right| \\
& \leq \int_{\{|u| \leq \alpha\}}(|u|+1)^{q-p+1}\left(\psi_{3}|\nabla u|^{p-1}+\psi_{1}|u|^{p-1}+\psi_{1}\right) \\
& \quad+\int_{\{|u|>\alpha\}}(\alpha+1)^{q-p}(|u|+1)\left(\psi_{3}|\nabla u|^{p-1}+\psi_{1}|u|^{p-1}+\psi_{1}\right) \\
& \leq \int_{\{|u| \leq \alpha\}} \psi_{3}\left(\frac{q}{p}\right)^{p-1}(|u|+1)^{\frac{q-p}{p}(p-1)}|\nabla u|^{p-1}(|u|+1)^{\frac{q}{p}}+2 \int_{\{|u| \leq \alpha\}} \psi_{1}(|u|+1)^{q} \\
& \quad+\int_{\{|u|>\alpha\}} \psi_{3}(\alpha+1)^{\frac{q-p}{p}(p-1)}|\nabla u|^{p-1}(\alpha+1)^{\frac{q-p}{p}}(|u|+1) \\
& \quad+2 \int_{\{|u|>\alpha\}} \psi_{1}(\alpha+1)^{q-p}(|u|+1)^{p} \\
& =\int_{\Omega} \psi_{3}\left|\nabla w_{\alpha}\right|^{p-1} w_{\alpha}+2 \int_{\Omega} \psi_{1} w_{\alpha}^{p}
\end{aligned}
$$

and

$$
\left|\int_{\partial \Omega} v_{\alpha} h(x, u)\right| \leq 2 \int_{\partial \Omega}\left|v_{\alpha}\right| \psi_{4}(|u|+1)^{p-1} \leq 2 \int_{\partial \Omega} \psi_{4} w_{\alpha}^{p}
$$

Using in addition that $u$ solves (4.2), we have shown that

$$
\begin{aligned}
0 & =\int_{\Omega} \nabla v_{\alpha} A(x, u, \nabla u)+\int_{\Omega} v_{\alpha} B(x, u, \nabla u)+\int_{\partial \Omega} v_{\alpha} h(x, u)+\omega \int_{\Omega} v_{\alpha} u \\
& \geq \frac{\nu}{2}\left(\frac{p}{q}\right)^{p} \int_{\Omega}\left|\nabla w_{\alpha}\right|^{p}-2(q-p+2) \int_{\Omega} \psi_{1} w_{\alpha}^{p}-\int_{\Omega} \psi_{3}\left|\nabla w_{\alpha}\right|^{p-1} w_{\alpha}-2 \int_{\partial \Omega} \psi_{4} w_{\alpha}^{p},
\end{aligned}
$$

i.e.,

$$
\left\|w_{\alpha}\right\|_{W^{1, p}(\Omega)}^{p} \leq c_{q} \int_{\Omega} \psi_{1} w_{\alpha}^{p}+c_{q} \int_{\Omega} \psi_{3}\left|\nabla w_{\alpha}\right|^{p-1} w_{\alpha}+c_{q} \int_{\partial \Omega} \psi_{4} w_{\alpha}^{p},
$$

where for simplicity we have assumed that $\psi_{1} \geq 1$ almost everywhere, which constitutes no loss of generality.

We now distinguish between the cases $p<N, p=N$ and $p>N$.

(a) Assume $p>N$. Then by [1, Theorem 5.8] there exists $\theta \in(0,1)$ such that

$$
\left\|w_{\alpha}\right\|_{L^{\infty}(\partial \Omega)} \leq\left\|w_{\alpha}\right\|_{L^{\infty}(\Omega)} \leq c\left\|w_{\alpha}\right\|_{W^{1, p}(\Omega)}^{\theta}\left\|w_{\alpha}\right\|_{L^{p}(\Omega)}^{1-\theta} .
$$


Hence

$$
\begin{aligned}
& \int_{\Omega} \psi_{1} w_{\alpha}^{p}+\int_{\Omega} \psi_{3}\left|\nabla w_{\alpha}\right|^{p-1} w_{\alpha}+\int_{\partial \Omega} \psi_{4} w_{\alpha}^{p} \\
& \leq\left\|\psi_{1}\right\|_{L^{1}(\Omega)}\left\|w_{\alpha}\right\|_{L^{\infty}(\Omega)}^{p}+\left\|\psi_{3}\right\|_{L^{p}(\Omega)}\left\|\left|\nabla w_{\alpha}\right|^{p-1}\right\|_{L^{\frac{p}{p-1}(\Omega)}}\left\|w_{\alpha}\right\|_{L^{\infty}(\Omega)} \\
& \quad+\left\|\psi_{4}\right\|_{L^{1}(\partial \Omega)}\left\|w_{\alpha}\right\|_{L^{\infty}(\partial \Omega)}^{p} \\
& \quad \leq c\left\|w_{\alpha}\right\|_{W^{1, p}(\Omega)}^{\theta p}\left\|w_{\alpha}\right\|_{L^{p}(\Omega)}^{(1-\theta) p}+c\left\|w_{\alpha}\right\|_{W^{1, p}(\Omega)}^{p-1+\theta}\left\|w_{\alpha}\right\|_{L^{p}(\Omega)}^{1-\theta} \\
& \leq \delta\left\|w_{\alpha}\right\|_{W^{1, p}(\Omega)}^{p}+c_{\delta}\left\|w_{\alpha}\right\|_{L^{p}(\Omega)}^{p}
\end{aligned}
$$

for every $\delta>0$ by Young's inequality. Picking $\delta>0$ small enough we obtain from this estimate and (4.8) that

$$
\left\|w_{\alpha}\right\|_{W^{1, p}(\Omega)}^{p} \leq c_{q}\left\|w_{\alpha}\right\|_{L^{p}(\Omega)}^{p} .
$$

This proves (4.3) since for $p=q$ we have $w_{\alpha}=|u|+1$ and $\left|\nabla w_{\alpha}\right|=|\nabla u|$, thus

$$
\|u\|_{W^{1, p}(\Omega)}^{p} \leq\left\|w_{\alpha}\right\|_{W^{1, p}(\Omega)}^{p} \leq c\left\|w_{\alpha}\right\|_{L^{p}(\Omega)}^{p} \leq c\|u\|_{L^{p}(\Omega)}^{p}+c
$$

Finally, estimate (4.3) implies (4.4), in this particular case even for $q=\infty$, by the Sobolev embedding theorems.

(b) Now assume $p=N$. Then by the interpolation inequality for Lebesgue spaces and the Sobolev embedding theorems we find for every $r \in[1, \infty)$ an exponent $\theta_{r} \in(0,1)$ such that

$$
\left\|w_{\alpha}\right\|_{L^{r}(\Omega)} \leq\left\|w_{\alpha}\right\|_{L^{2 r}(\Omega)}^{\theta_{r}}\left\|w_{\alpha}\right\|_{L^{1}(\Omega)}^{1-\theta_{r}} \leq c\left\|w_{\alpha}\right\|_{W^{1, p}(\Omega)}^{\theta_{r}}\left\|w_{\alpha}\right\|_{L^{p}(\Omega)}^{1-\theta_{r}} .
$$

Moreover, since the trace operator is compact from $W^{1, p}(\Omega)$ to $L^{r}(\partial \Omega)$ for every $r \in[1, \infty)$, we can estimate

$$
\left\|w_{\alpha}\right\|_{L^{r}(\partial \Omega)} \leq \delta\left\|w_{\alpha}\right\|_{W^{1, p}(\Omega)}+c_{\delta}\left\|w_{\alpha}\right\|_{L^{p}(\Omega)}
$$

for every $\delta>0$ by Lemma 4.1. Using these two estimates and Young's inequality, we obtain with certain exponents $\theta$ and $\theta^{\prime}$ in $(0,1)$ that

$$
\begin{aligned}
& \int_{\Omega} \psi_{1} w_{\alpha}^{p}+\int_{\Omega} \psi_{3}\left|\nabla w_{\alpha}\right|^{p-1} w_{\alpha}+\int_{\partial \Omega} \psi_{4} w_{\alpha}^{p} \\
& \leq\left\|\psi_{1}\right\|_{L^{\frac{p}{p-\varepsilon}}(\Omega)}\left\|w_{\alpha}^{p}\right\|_{L^{\frac{p}{\varepsilon}}(\Omega)}+\left\|\psi_{3}\right\|_{L^{\frac{p}{1-\varepsilon}}(\Omega)}\left\|\left|\nabla w_{\alpha}\right|^{p-1}\right\|_{L^{\frac{p}{p-1}}(\Omega)}\left\|w_{\alpha}\right\|_{L^{\frac{p}{\varepsilon}}(\Omega)} \\
& \quad+\left\|\psi_{4}\right\|_{L^{\frac{p-1}{p-1-\varepsilon}}(\partial \Omega)}\left\|w_{\alpha}^{p}\right\|_{L^{\frac{p-1}{\varepsilon}}}(\partial \Omega) \\
& \leq c\left\|w_{\alpha}\right\|_{W^{1, p}(\Omega)}^{p \theta}\left\|w_{\alpha}\right\|_{L^{p}(\Omega)}^{p(1-\theta)}+c\left\|w_{\alpha}\right\|_{W^{1, p}(\Omega)}^{p-1+\theta^{\prime}}\left\|w_{\alpha}\right\|_{L^{p}(\Omega)}^{1-\theta^{\prime}}+c\left\|_{\alpha}\right\|_{L^{\frac{p(p-1)}{\varepsilon}}}^{p}(\partial \Omega) \\
& \leq \delta\left\|w_{\alpha}\right\|_{W^{1, p}(\Omega)}^{p}+c_{\delta}\left\|w_{\alpha}\right\|_{L^{p}(\Omega)}^{p}
\end{aligned}
$$

for every $\delta>0$. As in the previous case $p>N$ this implies (4.3) and hence (4.4) by the Sobolev embedding theorems.

(c) Finally, assume $p<N$. Then

$$
\begin{aligned}
\int_{\Omega} \psi_{1} w_{\alpha}^{p} & \leq\left\|\psi_{1}\right\|_{L^{\frac{N}{p-\varepsilon}}(\Omega)}\left\|w_{\alpha}^{p-\varepsilon}\right\|_{L^{\frac{N p}{(N-p)(p-\varepsilon)}(\Omega)}}\left\|w_{\alpha}^{\varepsilon}\right\|_{L^{\frac{p}{\varepsilon}}(\Omega)} \\
& =c\left\|w_{\alpha}\right\|_{L^{\frac{N p}{N-p}}(\Omega)}^{p-\varepsilon}\left\|w_{\alpha}\right\|_{L^{p}(\Omega)}^{\varepsilon} \leq c\left\|w_{\alpha}\right\|_{W^{1, p}(\Omega)}^{p-\varepsilon}\left\|w_{\alpha}\right\|_{L^{p}(\Omega)}^{\varepsilon} \\
& \leq \delta\left\|w_{\alpha}\right\|_{W^{1, p}(\Omega)}^{p}+c_{\delta}\left\|w_{\alpha}\right\|_{L^{p}(\Omega)}^{p}
\end{aligned}
$$


for every $\delta>0$ by Young's inequality. Similarly,

$$
\begin{aligned}
& \int_{\Omega} \psi_{3}\left|\nabla w_{\alpha}\right|^{p-1} w_{\alpha} \\
& \quad \leq\|\psi\|_{L^{\frac{N}{1-\varepsilon}}(\Omega)}\left\|\left|\nabla w_{\alpha}\right|^{p-1}\right\|_{L^{\frac{p}{p-1}}(\Omega)}\left\|w_{\alpha}^{1-\varepsilon}\right\|_{L^{\frac{N p}{(N-p)(1-\varepsilon)}(\Omega)}}\left\|w_{\alpha}^{\varepsilon}\right\|_{L^{\frac{p}{\varepsilon}}(\Omega)} \\
& \quad \leq c\left\|w_{\alpha}\right\|_{W^{1, p}(\Omega)}^{p-1}\left\|w_{\alpha}\right\|_{L^{\frac{N p}{N-p}}(\Omega)}^{1-\varepsilon}\left\|w_{\alpha}\right\|_{L^{p}(\Omega)}^{\varepsilon} \leq c\left\|w_{\alpha}\right\|_{W^{1, p}(\Omega)}^{p-\varepsilon}\left\|w_{\alpha}\right\|_{L^{p}(\Omega)}^{\varepsilon} \\
& \quad \leq \delta\left\|w_{\alpha}\right\|_{W^{1, p}(\Omega)}^{p}+c_{\delta}\left\|w_{\alpha}\right\|_{L^{p}(\Omega)}^{p} .
\end{aligned}
$$

Moreover, since the trace operator is compact from $W^{1, p}(\Omega)$ to $L^{\frac{(N-1) p}{N-p+\varepsilon}}(\partial \Omega)$, we can estimate

$$
\left\|w_{\alpha}\right\|_{L^{\frac{(N-1) p}{N-p+\varepsilon}}(\partial \Omega)} \leq \delta\left\|w_{\alpha}\right\|_{W^{1, p}(\Omega)}+c_{\delta}\left\|w_{\alpha}\right\|_{L^{p}(\Omega)}
$$

for every $\delta>0$ by Lemma 4.1. Thus, picking $\delta>0$ small enough in the previous estimates, inequality (4.8) yields

$$
\left\|w_{\alpha}\right\|_{W^{1, p}(\Omega)}^{p} \leq c_{q}\left\|w_{\alpha}\right\|_{L^{p}(\Omega)}^{p} .
$$

As in the previous cases this implies (4.3).

Moreover, from (4.9) and the Sobolev embedding theorems we deduce that

$$
\left\|w_{\alpha}\right\|_{L^{\frac{N p}{N-p}}(\Omega)}^{p}+\left\|w_{\alpha}\right\|_{L^{\frac{(N-1) p}{N-p}}}^{p} \leq c \Omega\left\|w_{\alpha}\right\|_{W^{1, p}(\Omega)}^{p} \leq c_{q}\left\|w_{\alpha}\right\|_{L^{p}(\Omega)}^{p} .
$$

Since $w_{\alpha}$ approaches $(|u|+1)^{q / p}$ from below as $\alpha \rightarrow \infty$, the monotone convergence theorem implies that

$\left\|(|u|+1)^{q / p}\right\|_{L^{\frac{N p}{N-p}}(\Omega)}^{p}+\left\|(|u|+1)^{q / p}\right\|_{L^{\frac{(N-1) p}{N-p}}(\partial \Omega)}^{p} \leq c_{q}\left\|(|u|+1)^{q / p}\right\|_{L^{p}(\Omega)}$,

from which we can deduce that

$$
\|u\|_{L^{\frac{N q}{N-p}}(\Omega)}+\|u\|_{L^{\frac{(N-1) q}{N-p}}(\partial \Omega)} \leq c_{q}\|u\|_{L^{q}(\Omega)}+c_{q} .
$$

Since this estimate holds for all $q \in[1, \infty)$, defining

$$
q_{n}:=\left(\frac{N}{N-p}\right)^{n} p
$$

and iterating (4.10) we obtain that

$$
\begin{aligned}
\|u\|_{L^{q_{n}(\Omega)}}+\|u\|_{L^{\frac{N-1}{N} q_{n}}(\partial \Omega)} & =\|u\|_{L^{\frac{N q_{n-1}}{N-p}}(\Omega)}+\|u\|_{L^{\frac{(N-1) q_{n-1}}{N-p}}}(\partial \Omega) \\
& \leq c_{q_{n-1}}\|u\|_{L^{q_{n-1}}(\Omega)}+c_{q_{n-1}} \\
& \leq c_{q_{n-1}} c_{q_{n-2}}\|u\|_{L^{q_{n-2}(\Omega)}}+c_{q_{n-1}} c_{q_{n-2}}+c_{q_{n-1}} \\
& \leq \cdots \leq c_{n}\|u\|_{L^{q_{0}(\Omega)}}+c_{n}=c_{n}\|u\|_{L^{p}(\Omega)}+c_{n} .
\end{aligned}
$$

Since $q_{n} \rightarrow \infty$ as $n \rightarrow \infty$, this proves (4.4).

Remark 4.3. In the proof of Proposition 4.2 we silently passed over a subtlety that arises when deriving (4.8). A priori we can test the equation (4.2) only against functions in $W^{1, p}(\Omega) \cap L^{2}(\Omega)$. However, we had to test the equation against $v_{\alpha} \in$ $W^{1, p}(\Omega)$, but did not know a priori whether $v_{\alpha} \in L^{2}(\Omega)$. Still, since $\operatorname{sgn}\left(v_{\alpha}\right)=$ $\operatorname{sgn}(u)$ we can pick a sequence $\left(\eta_{n}\right)$ of functions in $W^{1, p}(\Omega) \cap L^{2}(\Omega)$ that converges to $v_{\alpha}$ in $W^{1, p}(\Omega)$ and such that the sequence $\left(\eta_{n} u\right)$ is pointwise monotonically 
increasing. Then it follows from the monotone convergence theorem that

$$
\begin{aligned}
0 & =\int_{\Omega} \nabla \eta_{n} A(x, u, \nabla u)+\int_{\Omega} \eta_{n} B(x, u, \nabla u)+\omega \int_{\Omega} \eta_{n} u+\int_{\partial \Omega} \eta_{n} h(x, u) \\
& \rightarrow \int_{\Omega} \nabla v_{\alpha} A(x, u, \nabla u)+\int_{\Omega} v_{\alpha} B(x, u, \nabla u)+\omega \int_{\Omega} v_{\alpha} u+\int_{\partial \Omega} v_{\alpha} h(x, u)
\end{aligned}
$$

as $n \rightarrow \infty$. Thus indeed equation (4.2) can be tested against $v_{\alpha}$, even before we know that $v_{\alpha} \in L^{2}(\Omega)$.

Now that we have $L^{q}$-bounds at our disposal, it is easy to deduce the Hölder continuity of solutions from the corresponding result concerning Neumann boundary conditions.

Theorem 4.4. Let $\Omega$ be a Lipschitz domain and assume (1.2) and (3.2). Then there exists $\alpha \in(0,1)$ such that every weak solution $u \in W^{1, p}(\Omega)$ of (4.1) with $\omega \geq 0$ is in $\mathrm{C}^{0, \alpha}(\Omega)$. Moreover, if $f, F$ and $g$ range over norm-bounded sets in their respective spaces, then the $\mathrm{C}^{0, \alpha}$-norms of the corresponding solutions $u$ remain bounded provided their norms in $L^{p}(\Omega)$ remain bounded.

Proof. Let $u \in W^{1, p}(\Omega)$ be a weak solution of (4.1). Then $u$ is a weak solution of

$$
\left\{\begin{aligned}
-\operatorname{div} \hat{A}(x, u, \nabla u)+\hat{B}(x, u, \nabla u)+\omega u=0 & \text { on } \Omega \\
\hat{A}(x, u, \nabla u) \cdot \nu+\hat{h}(x, u)=0 & \text { on } \partial \Omega
\end{aligned}\right.
$$

for $\hat{A}(x, u, z):=A(x, u, z)-F(x), \hat{B}(x, u, z):=B(x, u, z)-f(x)$ and $\hat{h}(x, u):=$ $h(x, u)-g(x)$. Assumption (3.2) ensures $\hat{A}, \hat{B}$ and $\hat{h}$ satisfy (1.2).

If $p>N$, then $u \in \mathrm{C}^{0, \alpha}(\Omega)$ by a Sobolev embedding theorem. The boundedness assertion about $u$ follows from Proposition 4.2 applied to the equation (4.11) since the constants in (4.3) depend only on upper bounds for the structure parameters of $\hat{A}, \hat{B}$ and $\hat{h}$, which in turn depend only on $A, B, h$ and upper bound for $f, F$ and $g$.

Now assume $p \leq N$. Pick $q \in[p, \infty)$ such that $q \geq \frac{2(N-1)(p-1)}{\varepsilon}$ and $q \geq \frac{N}{p-\varepsilon}$ with $\varepsilon>0$ as in the structure condition (1.2). We obtain from Proposition 4.2 applied to equation (4.11) that $u \in L^{q}(\Omega)$ and $\left.u\right|_{\partial \Omega} \in L^{q}(\partial \Omega)$ with a bound that depends only on bounds for the structure parameters of $\hat{A}, \hat{B}$ and $\hat{h}$. Thus

$$
h_{0}:=h(\cdot, u(\cdot)) \in L^{\frac{N-1}{p-1-\varepsilon / 2}}(\partial \Omega)
$$

by the structure assumption (1.2). Since $u$ is a weak solution of the Neumann problem

$$
\left\{\begin{aligned}
-\operatorname{div} A(x, u, \nabla u)+B(x, u, \nabla u) & =f(x)-\omega u(x)-(\operatorname{div} F)(x) & & \text { on } \Omega \\
A(x, u, \nabla u) \cdot \nu & =g(x)-h_{0}(x)+(F \cdot \nu)(x) & & \text { on } \partial \Omega,
\end{aligned}\right.
$$

we obtain that $u \in \mathrm{C}^{0, \alpha}(\Omega)$ with the same $\alpha \in(0,1)$ as in Theorem 3.7. The boundedness assertion about $u$ in $\mathrm{C}^{0, \alpha}(\Omega)$ follows from (3.8) and the observation that the right hand side of (4.12) can be estimated in terms of $A, B, h, \omega$ and upper bounds for $f, F$ and $h$.

Remark 4.5. Working with the full equation (4.1) instead of (4.2) in Proposition 4.2, we could have found an estimate like (3.8) also in the situation of Theorem (4.4). However, for our purposes nothing is gained by this more precise estimate, so for the sake of simplicity we avoided this additional effort.

Sometimes it is convenient to replace the functions on the right hand side of (4.1) by an arbitrary bounded linear functional on $W^{1, p}(\Omega)$. Therefore we formulate the regularity result of Theorem 4.4 also for such equations. 
Corollary 4.6. Let $\Omega \subset \mathbb{R}^{N}$ be a Lipschitz domain and assume (1.2). Let $q \in$ $[1, \infty)$ satisfy

$$
\begin{cases}q \leq \frac{N}{N-p+1} & \text { if } p<N \\ q<N & \text { if } p=N \\ q \leq p & \text { if } p>N\end{cases}
$$

and fix $\psi \in W^{1, q}(\Omega)^{\prime}$. Then every function $u \in W^{1, p}(\Omega)$ that satisfies

$$
\int_{\Omega} \nabla \eta A(x, u, \nabla u)+\int_{\Omega} \eta B(x, u, \nabla u)+\int_{\partial \Omega} \eta h(x, u)=\psi(\eta)
$$

for all $\eta \in \mathrm{C}^{\infty}(\bar{\Omega})$ is Hölder continuous on $\Omega$.

Proof. This is a direct consequence of the representation formula for functionals on Sobolev spaces [31, §4.3] and Theorem 4.4.

\section{Parabolic problems}

Let $\Omega \subset \mathbb{R}^{N}$ be a Lipschitz domain. We show that the parabolic problem

$$
\left\{\begin{aligned}
u_{t}(t, x)-\operatorname{div} a(x, \nabla u(t, x))+b(x, u(t, x)) & =0 & & t>0, x \in \Omega \\
a(x, \nabla u(t, x)) \cdot \nu+h(x, u(x)) & =0 & & t>0, x \in \partial \Omega \\
u(0, x) & =u_{0}(x) & & x \in \Omega
\end{aligned}\right.
$$

with Robin boundary conditions is well-posed in the space $\mathrm{C}(\bar{\Omega})$ under suitable conditions on $a, b$ and $h$. More precisely, we assume that $a: \Omega \times \mathbb{R}^{N} \rightarrow \mathbb{R}^{N}$, $b: \Omega \times \mathbb{R} \rightarrow \mathbb{R}$ and $h: \partial \Omega \times \mathbb{R} \rightarrow \mathbb{R}$ are measurable and continuous in the second argument. We also require that there exists $p \in(1, \infty)$ such that

$$
\left\{\begin{aligned}
z a(x, z) & \geq \nu|z|^{p}-\psi_{1}(x), & & |a(x, z)| \leq \mu|z|^{p-1}+\psi_{2}(x), \\
|b(x, u)| & \leq \psi_{1}(x)|u|^{p-1}+\psi_{1}(x), & & |h(x, u)| \leq \psi_{4}(x)|u|^{p-1}+\psi_{4}(x)
\end{aligned}\right.
$$

for all $x \in \Omega, u \in \mathbb{R}$ and $z \in \mathbb{R}^{N}$ with $\psi_{1}, \psi_{2}$ and $\psi_{4}$ as in (1.2), i.e., the functions $A(x, u, z):=a(x, z), B(x, u, z):=b(x, z)$ and $h$ satisfy (1.2). Moreover, we assume the monotonicity conditions

$$
\left\{\begin{aligned}
\left(z_{1}-z_{2}\right)\left(a\left(x, z_{1}\right)-a\left(x, z_{2}\right)\right) & \geq 0 \\
\left(u_{1}-u_{2}\right)\left(b\left(x, u_{1}\right)-b\left(x, u_{2}\right)\right) & \geq 0 \\
\left(u_{1}-u_{2}\right)\left(h\left(x, u_{1}\right)-h\left(x, u_{2}\right)\right) & \geq 0
\end{aligned}\right.
$$

for all $x \in \Omega, u_{1}, u_{2} \in \mathbb{R}$ and $z_{1}, z_{2} \in \mathbb{R}^{N}$.

In order to prove well-posedness in $\mathrm{C}(\bar{\Omega})$ we are going to show that the operator which is naturally associated with (5.1) is m-accretive on $\mathrm{C}(\bar{\Omega})$ and thus generates a semigroup on that space.

It is convenient to first introduce a version $\mathcal{A}$ of the operator associated with (5.1) acting from $V:=W^{1, p}(\Omega) \cap L^{2}(\Omega)$ into its $V^{\prime}$ and to study its properties. Later on we will turn our attention to its part in $\mathrm{C}(\bar{\Omega})$ in order to obtain the main result.

Proposition 5.1. The space $V:=W^{1, p}(\Omega) \cap L^{2}(\Omega)$ is a separable, reflexive Banach space for the norm $\|u\|_{V}:=\|u\|_{W^{1, p}(\Omega)}+\|u\|_{L^{2}(\Omega)}$. Under the assumptions (5.2) and (5.3), via

$$
\langle\mathcal{A} u, v\rangle:=\int_{\Omega} \nabla v a(x, \nabla u)+\int_{\Omega} v b(x, u)+\int_{\partial \Omega} v h(x, u) .
$$

we have defined a bounded, continuous, monotone operator $\mathcal{A}: V \rightarrow V^{\prime}$. 
Proof. We prove the assertions only for $p<N$ and only mention that the case $p \geq N$ is similar. Identifying $V$ with a closed subspace of the direct sum $W^{1, p}(\Omega) \oplus L^{2}(\Omega)$, we have proved the first claim. For $u$ and $v$ in $W^{1, p}(\Omega)$ we have by (5.2) that

$$
\begin{aligned}
\int_{\Omega}|\nabla v a(x, \nabla u)| & \leq\|\nabla v\|_{L^{p}(\Omega)}\left(\int_{\Omega}\left(\mu|\nabla u|^{p-1}+\psi_{2}\right)^{\frac{p}{p-1}}\right)^{\frac{p-1}{p}} \\
& \leq c\|v\|_{W^{1, p}(\Omega)}\left(\|\nabla u\|_{L^{p}(\Omega)}^{p-1}+\left\|\psi_{2}\right\|_{L^{\frac{p}{p-1}}(\Omega)}\right) \\
& \leq\left(c\|u\|_{V}^{p-1}+c\right)\|v\|_{V} .
\end{aligned}
$$

Similarly, using in addition the Sobolev embedding theorems, we obtain that

$$
\begin{aligned}
\int_{\Omega}|v b(x, u)| & \leq\|v\|_{L^{\frac{N p}{N-p}}(\Omega)}\left\|\psi_{1}\right\|_{L^{\frac{N}{p}}(\Omega)}\left\||u|^{p-1}+1\right\|_{L^{\frac{N p}{(N-p)(p-1)}}(\Omega)} \\
& \leq c\|v\|_{W^{1, p}(\Omega)}\left(c\|u\|_{W^{1, p}(\Omega)}^{p-1}+c\right) \leq\left(c\|u\|_{V}^{p-1}+c\right)\|v\|_{V}
\end{aligned}
$$

and

$$
\begin{aligned}
\int_{\partial \Omega}|v h(x, u)| & \leq\|v\|_{L^{\frac{(N-1) p}{N-p}}(\partial \Omega)}\left\|\psi_{4}\right\|_{L^{\frac{N-1}{p-1}}(\partial \Omega)}\left\||u|^{p-1}+1\right\|_{L^{\frac{(N-1) p}{(N-p)(p-1)}}(\partial \Omega)} \\
& \leq c\|v\|_{W^{1, p}(\Omega)}\left(c\|u\|_{W^{1, p}(\Omega)}^{p-1}+c\right) \leq\left(c\|u\|_{V}^{p-1}+c\right)\|v\|_{V} .
\end{aligned}
$$

Thus $\mathcal{A}$ is well-defined and $\|\mathcal{A} u\|_{V^{\prime}} \leq c\|u\|_{V}^{p-1}+c$ for all $u \in V$, proving that the operator $\mathcal{A}$ is bounded, i.e., that $\mathcal{A}$ maps bounded sets into bounded sets.

Now we show that $\mathcal{A}$ is continuous. To this end, let $\left(u_{n}\right)$ be a sequence in $V$ that converges to $u \in V$. Passing to a subsequence, we can assume that $\nabla u_{n} \rightarrow \nabla u$ pointwise and that $\left|\nabla u_{n}\right| \leq m$ for some function $m \in L^{p}(\Omega)$. Then $a\left(x, \nabla u_{n}(x)\right) \rightarrow$ $a(x, \nabla u(x))$ for almost every $x \in \Omega$ and

$$
\left|a\left(x, \nabla u_{n}\right)\right| \leq \mu m^{p-1}+\psi_{2} \in L^{\frac{p}{p-1}}(\Omega) .
$$

Hence $a\left(\cdot, \nabla u_{n}\right) \rightarrow a(\cdot, \nabla u)$ in $L^{\frac{p}{p-1}}(\Omega)$ by the dominated convergence theorem. An analogous reasoning yields that

$$
b\left(\cdot, u_{n}\right) \rightarrow b(\cdot, u) \text { in } L^{\frac{N p}{N p-N+p}}(\Omega) \quad \text { and } \quad h\left(\cdot, u_{n}\right) \rightarrow h(\cdot, u) \text { in } L^{\frac{(N-1) p}{N(p-1)}}(\partial \Omega) .
$$

Thus, given $\varepsilon>0$, we have

$$
\begin{aligned}
\left|\left\langle\mathcal{A} u_{n}-\mathcal{A} u, v\right\rangle\right| \leq \| & \nabla v\left\|_{L^{p}(\Omega)}\right\| a\left(\cdot, \nabla u_{n}\right)-a(\cdot, \nabla u) \|_{L^{\frac{p}{p-1}}(\Omega)} \\
& +\|v\|_{L^{\frac{N p}{N-p}}(\Omega)}\left\|b\left(\cdot, u_{n}\right)-b(\cdot, u)\right\|_{L^{\frac{N p}{N p-N+p}}(\Omega)} \\
& +\|v\|_{L^{\frac{(N-1) p}{N-p}}(\partial \Omega)}\left\|h\left(\cdot, u_{n}\right)-h(\cdot, u)\right\|_{L^{\frac{(N-1) p}{N(p-1)}}(\partial \Omega)} \\
\leq \varepsilon & v \|_{W^{1, p}(\Omega)}
\end{aligned}
$$

for $n \geq n_{0}(\varepsilon)$, which shows that $\mathcal{A} u_{n} \rightarrow \mathcal{A} u$ in $V^{\prime}$.

Finally, the monotonicity of $\mathcal{A}$, i.e., $\langle\mathcal{A} u-\mathcal{A} v, u-v\rangle \geq 0$ for all $u, v \in V$, is a trivial consequence of (5.3).

Next we show that $\mathcal{A}$ is bijective.

Proposition 5.2. Under the assumptions of Proposition 5.1, for every $\varphi \in V^{\prime}$ and every $\alpha>0$ there exists a unique function $u \in V$ such that

$$
(u \mid v)_{L^{2}(\Omega)}+\alpha\langle\mathcal{A} u, v\rangle=\varphi(v)
$$

for all $v \in V$. 
Proof. Define the operator $\mathcal{A}_{\alpha}: V \rightarrow V^{\prime}$ by

$$
\left\langle\mathcal{A}_{\alpha} u, v\right\rangle:=(u \mid v)_{L^{2}(\Omega)}+\alpha\langle\mathcal{A} u, v\rangle .
$$

From Proposition 5.1 we obtain that $\mathcal{A}_{\alpha}$ is bounded, continuous and monotone. From (5.2) we obtain that

$$
\int_{\Omega} \nabla u a(x, \nabla u) \geq \nu \int_{\Omega}|\nabla u|^{p}-\int_{\Omega} \psi_{1}=\nu\|\nabla u\|_{L^{p}(\Omega)}^{p}-c .
$$

for all $u \in V$. Moreover, (5.2) and (5.3) yield

$$
\begin{aligned}
\int_{\Omega} u b(x, u) & =\int_{\Omega}(u-0)(b(x, u)-b(x, 0))+\int_{\Omega} u b(x, 0) \geq-\int_{\Omega} \psi_{1}|u| \\
& \geq-\left\|\psi_{1}\right\|_{L^{\frac{N}{p}}(\Omega)}\|u\|_{L^{\frac{N}{N-p}}(\Omega)} \geq-c\|u\|_{W^{1, p}(\Omega)}
\end{aligned}
$$

for all $u \in V$, and analogously we see that $\int_{\Omega} u h(x, u) \geq-c\|u\|_{W^{1, p}(\Omega)}$. Combining the latter three estimates we have shown that

$$
\begin{aligned}
\left\langle\mathcal{A}_{\alpha} u, u\right\rangle & \geq\|u\|_{L^{2}(\Omega)}^{2}+\alpha \nu\|\nabla u\|_{L^{p}(\Omega)}^{p}-c\|u\|_{W^{1, p}(\Omega)} \\
& \geq \beta\left(\|u\|_{L^{2}(\Omega)}+\|\nabla u\|_{L^{p}(\Omega)}\right)^{q}-c\|u\|_{V}-c
\end{aligned}
$$

for $q:=\min \{2, p\}>1$ and some $\beta>0$.

From Lemma (4.1) we obtain that

$$
\|u\|_{L^{p}(\Omega)} \leq \delta\|u\|_{V}+c_{\delta}\|u\|_{L^{2}(\Omega)} \leq c \delta\|\nabla u\|_{L^{p}(\Omega)}+c \delta\|u\|_{L^{p}(\Omega)}+c_{\delta}\|u\|_{L^{2}(\Omega)}
$$

for all $u \in V$ with an arbitrary $\delta>0$. Picking $\delta>0$ small enough we deduce that

$$
\|u\|_{L^{p}(\Omega)} \leq c\|\nabla u\|_{L^{p}(\Omega)}+c\|u\|_{L^{2}(\Omega)} .
$$

Hence $\|u\|_{2, p}:=\|u\|_{L^{2}(\Omega)}+\|\nabla u\|_{L^{p}(\Omega)}$ is an equivalent norm on $V$. Thus from (5.6) we obtain that

$$
\liminf _{\|u\|_{V} \rightarrow \infty} \frac{\left\langle\mathcal{A}_{\alpha} u, u\right\rangle}{\|u\|_{V}} \geq \liminf _{\|u\|_{2, p} \rightarrow \infty} \frac{\beta\|u\|_{2, p}^{q}-c\|u\|_{2, p}-c}{\|u\|_{2, p}}=\infty
$$

and call (5.6) the coercivity of $\mathcal{A}_{\alpha}$. Since $\mathcal{A}_{\alpha}$ is also bounded, continuous and monotone, the operator is surjective by the Minty-Browder theorem [28, §II.2]. This means that (5.5) has a solution for every $\varphi \in V^{\prime}$. Moreover, if $\mathcal{A}_{\alpha} u_{1}=\mathcal{A}_{\alpha} u_{2}$ for two functions $u_{1}$ and $u_{2}$ in $V$, then by monotonicity of $\mathcal{A}$ we obtain that

$$
0=\left\langle\mathcal{A}_{\alpha} u_{1}-\mathcal{A}_{\alpha} u_{2}, u_{1}-u_{2}\right\rangle \geq\left(u_{1}-u_{2} \mid u_{1}-u_{2}\right)_{L^{2}(\Omega)}=\left\|u_{1}-u_{2}\right\|_{L^{2}(\Omega)}^{2},
$$

implying that $u_{1}=u_{2}$. Hence the solution of (5.5) is unique.

Our next step is to show that $(I+\alpha \mathcal{A})^{-1}$ is a contraction with respect to the norms of $L^{q}(\Omega)$ for $q \in[2, \infty]$. This is equivalent to saying that the part $\mathcal{A}$ in $L^{q}(\Omega)$ is accretive. Setting $q=\infty$ we thus obtain in particular that the part of $\mathcal{A}$ in $\mathrm{C}(\bar{\Omega})$ is accretive.

Proposition 5.3. Let (5.2) and (5.3) be satisfied. Let $f_{1}$ and $f_{2}$ be in $L^{q}(\Omega) \subset$ $L^{2}(\Omega), q \in[2, \infty]$ and define $\varphi_{i}(v):=\left(f_{i} \mid v\right)_{L^{2}(\Omega)}$ so that $\varphi_{i} \in V^{\prime}$. Then the unique solutions $u_{i}$ of (5.5) for the right hand sides $\varphi_{i}$ satisfy $\left\|u_{1}-u_{2}\right\|_{L^{q}(\Omega)} \leq$ $\left\|f_{1}-f_{2}\right\|_{L^{q}(\Omega)}$. Moreover, if $q>\frac{N}{p}$, then $u_{1}$ and $u_{2}$ are in $\mathrm{C}(\bar{\Omega})$.

Proof. Fix $q \in[2, \infty)$ and $k \geq 1$. Then by the chain rule [31, Theorem 2.2.2] the function

$$
v_{k}:=\left(\left|u_{1}-u_{2}\right| \wedge k\right)^{q-2}\left(u_{1}-u_{2}\right)
$$

where $x \wedge y$ denotes the minimum of $x$ and $y$, is in $V$ with weak derivative $\nabla v_{k}=(q-1)\left|u_{1}-u_{2}\right|^{q-2}\left(\nabla u_{1}-\nabla u_{2}\right) \mathbb{1}_{\left\{\left|u_{1}-u_{2}\right| \leq k\right\}}+k^{q-2}\left(\nabla u_{1}-\nabla u_{2}\right) \mathbb{1}_{\left\{\left|u_{1}-u_{2}\right|>k\right\}}$. 
Since at each point $v_{k}$ is a positive multiple of $u_{1}-u_{2}$ and $\nabla v_{k}$ is a positive multiple of $\nabla u_{1}-\nabla u_{2}$, we deduce from (5.3) that

$$
\left\langle\mathcal{A} u_{1}-\mathcal{A} u_{2}, v_{k}\right\rangle \geq 0 .
$$

Hence with $\mathcal{A}_{\alpha}$ as in the proof of Proposition 5.2 we obtain that

$$
\begin{gathered}
\int_{\Omega}\left(\left|u_{1}-u_{2}\right| \wedge k\right)^{q-2}\left|u_{1}-u_{2}\right|^{2}=\left(u_{1}-u_{2} \mid v_{k}\right)_{L^{2}(\Omega)} \leq\left\langle\mathcal{A}_{\alpha} u_{1}-\mathcal{A}_{\alpha} u_{2}, v_{k}\right\rangle \\
\quad=\varphi_{1}\left(v_{k}\right)-\varphi_{2}\left(v_{k}\right) \leq\left\|f_{1}-f_{2}\right\|_{L^{q}(\Omega)}\left\|v_{k}\right\|_{L^{\frac{q}{q-1}}(\Omega)} \\
\quad \leq\left\|f_{1}-f_{2}\right\|_{L^{q}(\Omega)}\left(\int_{\Omega}\left(\left|u_{1}-u_{2}\right| \wedge k\right)^{q-2}\left|u_{1}-u_{2}\right|^{2}\right)^{\frac{q-1}{q}}
\end{gathered}
$$

Dividing in this equation and afterwards letting $k$ tend to infinity, we obtain from the monotone convergence theorem that $\left\|u_{1}-u_{2}\right\|_{L^{q}(\Omega)} \leq\left\|f_{1}-f_{2}\right\|_{L^{q}(\Omega)}$ for every $q \in[2, \infty)$. If $f_{1}$ and $f_{2}$ are in $L^{\infty}(\Omega)$, we pass to the limit $q \rightarrow \infty$ and obtain that $\left\|u_{1}-u_{2}\right\|_{L^{\infty}(\Omega)} \leq\left\|f_{1}-f_{2}\right\|_{L^{\infty}(\Omega)}$.

Now assume $q>\frac{N}{p}$. By definition, the function $u_{i}$ is a weak solution of

$$
\left\{\begin{aligned}
-\operatorname{div} a(x, \nabla u)+b(x, u) & =f_{i} & & \text { on } \Omega \\
a(x, \nabla u) \cdot \nu+h(x, u) & =0 & & \text { on } \partial \Omega .
\end{aligned}\right.
$$

Hence $u_{i} \in \mathrm{C}^{0, \alpha}(\Omega) \subset \mathrm{C}(\bar{\Omega})$ by Theorem 4.4 .

Regard $\mathrm{C}(\bar{\Omega})$ as a subspace of $V^{\prime}$ by identifying a function $f \in \mathrm{C}(\bar{\Omega})$ with the linear functional $v \mapsto(f \mid v)_{L^{2}(\Omega)}$. Then the part $\mathcal{A}^{c}$ of $\mathcal{A}$ in $\mathrm{C}(\bar{\Omega})$ is the restriction of $\mathcal{A}$ to

$$
D\left(\mathcal{A}^{c}\right):=\{u \in V \cap \mathrm{C}(\bar{\Omega}): \mathcal{A} u \in \mathrm{C}(\bar{\Omega})\} .
$$

We regard $\mathcal{A}^{c}$ as a non-linear (and single-valued) operator on $\mathrm{C}(\bar{\Omega})$. Proposition 5.3 implies that $\mathcal{A}^{c}$ is m-accretive, i.e., for all $\alpha>0$ the operator $I+\alpha \mathcal{A}^{c}: D\left(\mathcal{A}^{c}\right) \rightarrow$ $\mathrm{C}(\bar{\Omega})$ is bijective and $\left(I+\alpha \mathcal{A}^{c}\right)^{-1}$ is a contraction on $\mathrm{C}(\bar{\Omega})$. We now show that $\mathcal{A}^{c}$ is densely defined.

Proposition 5.4. Under assumptions (5.2) and (5.3) the set $D\left(\mathcal{A}^{c}\right)$ is dense in $\mathrm{C}(\bar{\Omega})$.

Proof. We give the proof only for $p<N$ and only mention that the case $p \geq N$ can be treated analogously. First assume that $u \in \mathrm{C}^{\infty}(\bar{\Omega})$, so in particular $u \in V$. Then $\varphi:=\mathcal{A}_{1} u \in V^{\prime}$, where $\mathcal{A}_{1}$ is defined as in the proof of Proposition 5.2. More precisely,

$$
\begin{aligned}
|a(x, \nabla u)| & \leq \mu\|\nabla u\|_{L^{\infty}(\Omega)}^{p-1}+\psi_{2} \leq c_{u}+\psi_{2} \\
|b(x, u)| & \leq \psi_{1}\left(\|u\|_{L^{\infty}(\Omega)}^{p-1}+1\right) \leq c_{u} \psi_{1} \\
|h(x, u)| & \leq \psi_{4}\left(\|u\|_{L^{\infty}(\Omega)}^{p-1}+1\right) \leq c_{u} \psi_{4}
\end{aligned}
$$

and hence

$$
\begin{aligned}
\varphi(v)= & \int_{\Omega} v u+\int_{\Omega} \nabla v a(x, \nabla u)+\int_{\Omega} v b(x, u)+\int_{\partial \Omega} v h(x, u) \\
\leq & c_{u}\|v\|_{L^{1}(\Omega)}+\|\nabla v\|_{L^{\frac{N}{N-p+1}}(\Omega)}\left\|c_{u}+\psi_{2}\right\|_{L^{\frac{N}{p-1}}(\Omega)} \\
& \quad+c_{u}\|v\|_{L^{\frac{N}{N-p}}(\Omega)}\left\|\psi_{1}\right\|_{L^{\frac{N}{p}}(\Omega)}+c_{u}\|v\|_{L^{\frac{N-1}{N-p}}(\partial \Omega)}\left\|\psi_{4}\right\|_{L^{\frac{N-1}{p-1}}(\partial \Omega)} \\
\leq & c_{u}\|v\|_{W^{1, \frac{N}{N-p+1}}(\Omega)}
\end{aligned}
$$


for all $v \in V$. Thus $\psi$ extends to a bounded functional on $W^{1, \frac{N}{N-p+1}}(\Omega)$. Consequently, there exist $k \in L^{\frac{N}{p-1}}(\Omega)$ and $K \in L^{\frac{N}{p-1}}\left(\Omega ; \mathbb{R}^{N}\right)$ such that

$$
\varphi(v)=\int_{\Omega} v k+\int_{\Omega} \nabla v K
$$

for all $v \in V$, see [31, §4.3]. Pick sequences $k_{n} \in \mathrm{C}_{c}^{\infty}(\Omega)$ and $K_{n} \in \mathrm{C}_{c}^{\infty}\left(\Omega ; \mathbb{R}^{N}\right)$ such that $k_{n} \rightarrow k$ and $K_{n} \rightarrow K$ in the $L^{\frac{N}{p-1}}$-norm. Then $f_{n}:=k_{n}-\operatorname{div} K_{n}$ is in $\mathrm{C}_{c}^{\infty}(\Omega) \subset \mathrm{C}(\bar{\Omega})$. Thus by Propositions 5.2 and the additional claim in (5.3) there exists $u_{n} \in D\left(\mathcal{A}^{c}\right)$ such that $\left(I+\mathcal{A}^{c}\right) u_{n}=f_{n}$.

Define $\varphi_{n}(v):=\left(f_{n} \mid v\right)_{L^{2}(\Omega)}$, which can equivalently be written as $\varphi_{n}:=\mathcal{A}_{1} u_{n}$. Then

$$
\begin{aligned}
\left|\varphi_{n}(v)-\varphi(v)\right| & \leq \int_{\Omega}|v|\left|k_{n}-k\right|+\int_{\Omega}|\nabla v|\left|K_{n}-K\right| \\
& \leq\|v\|_{W^{1, p}(\Omega)}\left(\left\|k_{n}-k\right\|_{L^{\frac{p}{p-1}}(\Omega)}+\left\|K_{n}-K\right\|_{L^{\frac{p}{p-1}}(\Omega)}\right) .
\end{aligned}
$$

This shows that $\varphi_{n} \rightarrow \varphi$ in $V^{\prime}$. In particular we see that $\left(\varphi_{n}\right)$ is bounded in $V^{\prime}$, which implies that

$$
\left\langle\mathcal{A}_{1} u_{n}, u_{n}\right\rangle=\varphi_{n}\left(u_{n}\right) \leq c_{u}\left\|u_{n}\right\|_{V} .
$$

By (5.7) this implies that $\left(u_{n}\right)$ is bounded in $V$. Thus passing to a subsequence we can assume that $\left(u_{n}\right)$ converges weakly to some $\tilde{u} \in V$.

By Minty's theorem [28, Proposition II.2.2] we have

$$
\left\langle\mathcal{A}_{1} v-\varphi_{n}, v-u_{n}\right\rangle \geq 0
$$

for all $v \in V$. Since $\varphi_{n} \rightarrow \varphi$ in $V^{\prime}$ and $u_{n} \rightarrow \tilde{u}$ in $V$ we obtain by taking limits that

$$
\left\langle\mathcal{A}_{1} v-\varphi, v-\tilde{u}\right\rangle \geq 0
$$

for all $v \in V$. Using Minty's theorem once again we deduce from this that $\mathcal{A}_{1} \tilde{u}=$ $\varphi=\mathcal{A}_{1} u$. By the uniqueness assertion of Proposition 5.2 this implies $\tilde{u}=u$. Thus we have shown that $u_{n} \rightarrow u$ in $V$.

We have seen that $\left(u_{n}\right)$ is bounded in $V$ and hence in particular in $L^{p}(\Omega)$. Since by construction $u_{n}$ is a weak solution of

$$
\left\{\begin{aligned}
-\operatorname{div} a\left(x, \nabla u_{n}\right)+b\left(x, u_{n}\right) & =k_{n}-\operatorname{div} K_{n} & & \text { on } \Omega \\
a\left(x, \nabla u_{n}\right) \cdot \nu+h\left(x, u_{n}\right) & =K_{n} \cdot \nu & & \text { on } \partial \Omega,
\end{aligned}\right.
$$

we obtain from Theorem 4.4 that $\left(u_{n}\right)$ is bounded in $\mathrm{C}^{0, \alpha}(\Omega)$. Exploiting compactness we can assume after passing to a subsequence that $\left(u_{n}\right)$ has a limit in $\mathrm{C}(\bar{\Omega})$. Since $u_{n} \rightarrow u$ in $V$, this limit is $u$. Hence we have shown that for each $u \in \mathrm{C}^{\infty}(\bar{\Omega})$ there exists a sequence $u_{n} \in D\left(\mathcal{A}^{c}\right)$ such that $u_{n} \rightarrow u$ in $\mathrm{C}(\bar{\Omega})$. This proves that $\mathrm{C}^{\infty}(\bar{\Omega})$ is contained in the closure of $D\left(\mathcal{A}^{c}\right)$ in $\mathrm{C}(\bar{\Omega})$. Since $\mathrm{C}^{\infty}(\bar{\Omega})$ is dense in $\mathrm{C}(\bar{\Omega})$, we have shown that $D\left(\mathcal{A}^{c}\right)$ is dense in $\mathrm{C}(\bar{\Omega})$.

In order to state the main result of this section it is convenient to introduce the notion of a non-linear contraction $\mathrm{C}_{0}$-semigroup. The definition is consistent with the linear case, i.e., a family of linear operators on a Banach space $X$ is a non-linear contraction $\mathrm{C}_{0}$-semigroup if and only if it is a linear contraction $\mathrm{C}_{0}$-semigroup in the usual sense of for example [19].

Definition 5.5. Let $X$ be a Banach space and let $\mathcal{B}: X \supset D(\mathcal{B}) \rightarrow X$ be an m-accretive operator on $X$, i.e., for all $\alpha>0$ the operator $I+\alpha \mathcal{B}: D(\mathcal{B}) \rightarrow X$ is bijective with $(I+\alpha \mathcal{B})^{-1}: X \rightarrow X$ being a contraction. Then by the CrandallLiggett theorem [28, §IV.8] the limit $S(t) u_{0}=\lim _{n \rightarrow \infty}\left(I+\frac{t}{n} \mathcal{B}\right)^{-n} u_{0}$ exists for $u_{0} \in C:=\overline{D(\mathcal{B})}$ and $t \geq 0$ and the mappings $S(t)$ satisfy

(i) $S(t): C \rightarrow C$ is contractive for every $t \geq 0$; 
(ii) $S(0)=\operatorname{id}_{C}$;

(iii) $S(t+s)=S(t) \circ S(s)$ for all $t, s \geq 0$;

(iv) $t \mapsto S(t) u_{0}$ is continuous for all $u_{0} \in C$.

We say that $(S(t))_{t \geq 0}$ is a non-linear contraction $\mathrm{C}_{0}$-semigroup on $C$ and call $-\mathcal{B}$ its generator.

The following remark about non-linear contraction $\mathrm{C}_{0}$-semigroups and their generators justifies that we regard the trajectories of a non-linear contraction $\mathrm{C}_{0^{-}}$ semigroup with generator $-\mathcal{B}$ as the unique solutions of the problem $u^{\prime}(t)+\mathcal{B} u(t)=$ 0 . For the definition of a $\mathrm{C}^{0}$-solution, which is frequently also called mild solution in the literature, and proofs of the following fact we refer to [28, §IV.3 and §IV.8] or [7].

Remark 5.6. If $\mathcal{B}$ is $\mathrm{m}$-accretive and $(S(t))_{t>0}$ is the semigroup generated by $-\mathcal{B}$, then for each $u_{0} \in C:=\overline{D(\mathcal{B})}$ and $T>0$ the unique $\mathrm{C}^{0}$-solution of $u^{\prime}(t)+\mathcal{B} u(t)=0$, $u(0)=u_{0}$ on $[0, T]$ is given by $u(t)=S(t) u_{0}$.

Moreover, if $X$ is a Hilbert space and $u_{0} \in D(\mathcal{B})$, then this unique solution $u$ is Lipschitz continuous, $u(t) \in D(\mathcal{B})$ for all $t \geq 0$ and $u^{\prime}(t)+\mathcal{B} u(t)=0$ for almost every $t>0$. In this situation we say that $u$ is a strong solution of $u^{\prime}(t)+\mathcal{B} u(t)=0$.

Now regard $L^{2}(\Omega)$ as a subspace of $V$. Then the part $\mathcal{A}^{H}$ of $\mathcal{A}$ in $L^{2}(\Omega)$, i.e., the restriction of $\mathcal{A}$ to

$$
D\left(\mathcal{A}^{H}\right):=\left\{u \in V: \mathcal{A} u \in L^{2}(\Omega)\right\},
$$

acts as an m-accretive operator on $L^{2}(\Omega)$ by Propositions 5.2 and 5.3 . Moreover, the set $D\left(\mathcal{A}^{H}\right)$ is dense in $L^{2}(\Omega)$ by Proposition 5.4 since $D\left(\mathcal{A}^{c}\right) \subset D\left(\mathcal{A}^{H}\right)$ and $\mathrm{C}(\bar{\Omega})$ is continuously and densely embedded into $L^{2}(\Omega)$. Hence $-\mathcal{A}^{H}$ generates a non-linear contraction $\mathrm{C}_{0}$-semigroup $(S(t))_{t>0}$ on $L^{2}(\Omega)$. In view of Remark 5.6 we can justly call $u(t, x):=\left(S(t) u_{0}\right)(x)$ the unique solution of (5.1) for $u_{0} \in L^{2}(\Omega)$, and we refer to this solution as the $L^{2}$-solution of (5.1). The following is our main result.

Theorem 5.7. Assume (5.2) and (5.3). Then $\mathcal{A}^{c}$ is m-accretive on $\mathrm{C}(\bar{\Omega})$, the semigroup generated by $-\mathcal{A}^{c}$ being the restriction of $(S(t))_{t>0}$ to $\mathrm{C}(\bar{\Omega})$. Thus for $u_{0} \in \mathrm{C}(\bar{\Omega})$ the unique $L^{2}$-solution $u$ of (5.1) is continuous on the closed parabolic cylinder $[0, \infty) \times \bar{\Omega}$, i.e., continuous up to the parabolic boundary.

Proof. By Propositions 5.2 and 5.3 the operator $\mathcal{A}^{c}$ is m-accretive. Hence $-\mathcal{A}^{c}$ generates a non-linear contraction $\mathrm{C}_{0}$-semigroup $\left(S^{c}(t)\right)_{t \geq 0}$ on $\mathrm{C}(\bar{\Omega})$, see Definition 5.5 and Proposition (5.4). Since $\left(I+\alpha \mathcal{A}^{c}\right)^{-1}$ is the restriction of $\left(I+\alpha \mathcal{A}^{H}\right)^{-1}$ to $\mathrm{C}(\bar{\Omega})$, see the additional assertion in Proposition 5.3, the operator $S^{c}(t)$ is the restriction of $S(t)$ to $\mathrm{C}(\bar{\Omega})$. Thus $t \mapsto S^{H}(t) u_{0}$ is continuous as a function with values in $\mathrm{C}(\bar{\Omega})$ provided that $u_{0} \in \mathrm{C}(\bar{\Omega})$. In this case $u(t, x):=\left(S(t) u_{0}\right)(x)$ is jointly continuous with respect to $t \geq 0$ and $x \in \bar{\Omega}$.

Remark 5.8. Since $\mathcal{A}^{c}$ is m-accretive, we have a unique solution $u \in \mathrm{C}([0, \infty) \times \bar{\Omega})$ even for the inhomogeneous problem

$$
\left\{\begin{aligned}
u_{t}(t, x)-\operatorname{div} a(x, \nabla u(t, x))+b(x, u(t, x)) & =f(t, x) & & t>0, x \in \Omega \\
a(x, \nabla u(t, x)) \cdot \nu+h(x, u(x) & =0 & & t>0, x \in \partial \Omega \\
u(0, x) & =u_{0}(x) & & x \in \Omega
\end{aligned}\right.
$$

whenever $u_{0} \in \mathrm{C}(\bar{\Omega})$ and $f \in L_{\text {loc }}^{1}([0, \infty) ; \mathrm{C}(\bar{\Omega}))$, see [28, Corolllary IV.8.4].

Let us finally look at a class of examples that satisfy the conditions (5.2) and (5.3). Since in the diffusion equation (5.1) the function $a(x, \nabla u)$ is the flux, it is natural to 
assume that it points into the direction of steepest descent, i.e., into the direction of $\nabla u$. If we assume in addition that the magnitude of the flux depends only on the steepness of $u$ and possibly on the location $x \in \Omega$, i.e., if we assume that $a(x, z)=m(x,|z|) z$, then we have a simple criterion to check condition (5.3).

Lemma 5.9. Assume that $a(x, z)=m(x,|z|) z$ for a measurable function $m: \mathbb{R} \times$ $\mathbb{R}_{+} \rightarrow \mathbb{R}_{+}$. Then the first condition in (5.3) is satisfied if and only if $y \mapsto m(x, y) y$ is nondecreasing for every $x \in \Omega$. Similar assertions hold for $b$ and $h$.

Proof. For all $x \in \Omega$ and all $z_{1}$ and $z_{2}$ in $\mathbb{R}^{N}$ we have

$$
\begin{aligned}
\left(z_{1}-\right. & \left.z_{2}\right)\left(a\left(x, z_{1}\right)-a\left(x, z_{2}\right)\right) \\
& =m\left(x,\left|z_{1}\right|\right)\left|z_{1}\right|^{2}-\left(m\left(x,\left|z_{1}\right|\right)+m\left(x,\left|z_{2}\right|\right)\right) z_{1} \cdot z_{2}+m\left(x,\left|z_{2}\right|\right)\left|z_{2}\right|^{2} \\
& \geq m\left(x,\left|z_{1}\right|\right)\left|z_{1}\right|^{2}-\left(m\left(x,\left|z_{1}\right|\right)+m\left(x,\left|z_{2}\right|\right)\right)\left|z_{1}\right|\left|z_{2}\right|+m\left(x,\left|z_{2}\right|\right)\left|z_{2}\right|^{2} \\
& =\left(\left|z_{1}\right|-\left|z_{2}\right|\right)\left(m\left(x,\left|z_{1}\right|\right)\left|z_{1}\right|-m\left(x,\left|z_{2}\right|\right)\left|z_{2}\right|\right)
\end{aligned}
$$

with equality if $z_{1}$ and $z_{2}$ point into the same direction.

If $y \mapsto m(x, y) y$ is nondecreasing, then both factors in the last expression have the same sign. Thus the product is nonnegative and the first condition in (5.3) is fulfilled.

Conversely, if the first condition in (5.3) is fulfilled, then by choosing $z_{2}$ to be a positive multiple of $z_{1}$, we see that

$$
(a-b)(m(x, a) a-m(x, b) a) \geq 0
$$

for all $a, b>0$, which implies that $y \mapsto m(x, y) y$ is nondecreasing.

As a consequence of Lemma 5.9 we see that an important and commonly encountered class of equations of $p$-Laplace-type satisfy conditions (5.2) and (5.3). For simplicity we consider only examples with $p<N$, but similar assertions hold true for $p \geq N$ with slightly different integrability assumptions on the coefficients.

Example 5.10. Let $p \in(1, N)$. Set $a(x, z):=a_{0}(x)\left(s+|z|^{p-2}\right) z$ or $a(x, z):=$ $a_{0}(x)\left(s^{2}+|z|^{2}\right)^{\frac{p-2}{2}} z$ with a constant $s \geq 0$ and a measurable function $a_{0}: \Omega \rightarrow[\nu, \mu]$, where $0<\nu \leq \mu$. Set $b(x, u):=b_{0}(x)|u|^{p-2} u$ and $h(x, u):=h_{0}(x)|u|^{p-2} u$ with nonnegative measurable functions $b_{0} \in L^{\frac{N}{p-\varepsilon}}(\Omega)$ and $h_{0} \in L^{\frac{N-1}{p-1-\varepsilon}}(\partial \Omega)$ for some $\varepsilon>0$. Then the assumption in (5.2) and (5.3) are satisfied and thus Theorem 5.7 applies. In the special case $s=0$ and $a_{0}(x)=1$ for all $x \in \Omega$ we obtain that the equation

$$
\left\{\begin{aligned}
u_{t}(t, x)-\Delta_{p} u(t, x)+b_{0}(x)|u|^{p-2} u & =f(t, x) & & t>0, x \in \Omega \\
|\nabla u(t, x)|^{p-2} \frac{\partial u(t, x)}{\partial \nu}+h_{0}(x)|u|^{p-2} u & =0 & & t>0, x \in \partial \Omega \\
u(0, x) & =u_{0}(x) & & x \in \Omega
\end{aligned}\right.
$$

has a unique $\mathrm{C}^{0}$-solution $u \in \mathrm{C}([0, \infty) \times \bar{\Omega})$.

The strategy of this section applies also to certain dynamic boundary conditions, which are often called Wentzell-Robin boundary conditions, if we carry out the arguments on a suitable product space, confer also [2] where this idea was originally introduced to the literature for linear equations. More precisely, we consider the equation

$$
\left\{\begin{aligned}
u_{t}(t, x)-\operatorname{div} a(x, \nabla u(t, x))+b(x, u(t, x)) & =0 & & t>0, x \in \Omega \\
\beta u_{t}(t, x)+a(x, \nabla u(t, x)) \cdot \nu+h(x, u(t, x)) & =0 & & t>0, x \in \partial \Omega \\
u(0, x) & =u_{0}(x) & & x \in \Omega .
\end{aligned}\right.
$$


At least formally, we can use the first line in (5.8) to express $u_{t}$ in terms of $u$ in the second line. Then we arrive at what is classically called Wentzell-Robin boundary conditions.

We can show well-posedness of (5.8) in the space of continuous functions. Since the arguments are very similar to what we did before, we only sketch the proof of the following theorem, but see [25] for a detailed proof in the linear case.

Theorem 5.11. Assume (5.2) and (5.3). Let $\beta: \partial \Omega \rightarrow \mathbb{R}$ be measurable and such that

$$
0<\underset{\partial \Omega}{\operatorname{essinf}} \beta \leq \underset{\partial \Omega}{\operatorname{ess} \sup } \beta<\infty .
$$

For $u_{0} \in \mathrm{C}(\bar{\Omega})$, problem (5.8) has a unique solution. This solution is continuous on the parabolic cylinder $[0, \infty) \times \bar{\Omega}$.

Proof. Define the reflexive, separable Banach space

$$
\mathcal{V}:=\left\{\left(u,\left.u\right|_{\partial \Omega}\right): u \in W^{1, p}(\Omega) \cap L^{2}(\Omega),\left.u\right|_{\partial \Omega} \in L^{2}(\partial \Omega)\right\}
$$

and let $\mathcal{A}_{W}: \mathcal{V} \rightarrow \mathcal{V}^{\prime}$ be defined by the formal expression (5.4). The proof of Proposition 5.1 shows that $\mathcal{A}_{W}$ is bounded, continuous and monotone. For $q \in$ $[2, \infty]$ consider $L^{q}(\Omega) \oplus_{q} L^{q}(\partial \Omega)$, which for $q \in[2, \infty)$ is equipped with the norm given by

whereas for $q=\infty$ we set

$$
\|(u, g)\|_{L^{q}(\Omega) \oplus_{q} L^{q}(\partial \Omega)}^{q}:=\|u\|_{L^{q}(\Omega)}^{q}+\|g\|_{L^{q}(\partial \Omega ; \beta \mathrm{d} \sigma)}^{q},
$$

$$
\|(u, g)\|_{L^{\infty}(\Omega) \oplus_{\infty} L^{\infty}(\partial \Omega)}:=\|u\|_{L^{\infty}(\Omega)}+\|g\|_{L^{\infty}(\partial \Omega ; \beta \mathrm{d} \sigma)} .
$$

Here $\sigma$ denotes the surface measure on $\partial \Omega$, i.e., the $(N-1)$-dimensional Hausdorff measure. Set $\mathcal{H}:=L^{2}(\Omega) \oplus L^{2}(\partial \Omega)$, and equip $\mathcal{C}:=\left\{\left(u,\left.u\right|_{\partial \Omega}\right): u \in \mathrm{C}(\bar{\Omega})\right\}$ with the norm of $L^{\infty}(\Omega) \oplus_{\infty} L^{\infty}(\partial \Omega)$. Then both of these spaces are subspaces of $V^{\prime}$ via

$$
\left\langle(u, g),\left(v,\left.v\right|_{\partial \Omega}\right)\right\rangle_{V^{\prime}, V}:=(u \mid v)_{L^{2}(\Omega)}+(g \mid v)_{L^{2}(\partial \Omega ; \beta \mathrm{d} \sigma)}
$$

for $v \in V$ and $(u, g) \in \mathcal{H}$ or $(u, g) \in \mathcal{C}$, respectively. We consider the parts $\mathcal{A}_{W}^{\mathcal{H}}$ and $\mathcal{A}_{W}^{\mathcal{C}}$ of $\mathcal{A}_{W}$ in $\mathcal{H}$ and $\mathcal{C}$, respectively, with domains

$$
\begin{aligned}
& D\left(\mathcal{A}_{W}^{\mathcal{H}}\right):=\left\{u \in \mathcal{V}: \mathcal{A}_{W} u \in \mathcal{H}\right\} \\
& D\left(\mathcal{A}_{W}^{\mathcal{C}}\right):=\left\{u \in \mathcal{V} \cap \mathcal{C}: \mathcal{A}_{W} u \in \mathcal{C}\right\}
\end{aligned}
$$

Then similar arguments as in the proof of Propositions 5.2 and 5.3 show that $\mathcal{A}_{W}^{\mathcal{H}}$ and $\mathcal{A}_{W}^{\mathcal{C}}$ are m-accretive on $\mathcal{H}$ and $\mathcal{C}$, respectively. Let $(\mathcal{S}(t))_{t \geq 0}$ be the semigroup generated by $-\mathcal{A}_{W}^{\mathcal{H}}$. If $U_{0} \in D\left(\mathcal{A}_{W}^{\mathcal{H}}\right)$ and $U(t):=\mathcal{S}(t) U_{0}$, then $U^{\prime}(t)+\mathcal{A}_{W}^{\mathcal{H}} U(t)=0$ for almost every $t \geq 0$. Writing $U(t)=\left(u(t),\left.u(t)\right|_{\partial \Omega}\right)$ this means that

$$
\begin{aligned}
& \int_{\Omega} \nabla \eta a(x, \nabla u(t))+\int_{\Omega} \eta b(x, u(t))+\int_{\partial \Omega} \eta h(x, u) \\
& \quad=\left\langle\mathcal{A}_{W}^{\mathcal{H}},\left(\eta,\left.\eta\right|_{\partial \Omega}\right)\right\rangle=\left(u^{\prime}(t) \mid\left(\eta,\left.\eta\right|_{\partial \Omega}\right)\right)_{\mathcal{H}}=-\int_{\Omega} \eta u^{\prime}(t)-\int_{\partial \Omega} \eta u^{\prime}(t) \beta
\end{aligned}
$$

for all $\eta \in \mathrm{C}^{\infty}(\bar{\Omega}) \subset \mathcal{V}$ and almost every $t \geq 0$. Hence for almost every $t \geq 0$, the function $u(t) \in W^{1, p}(\Omega)$ is a weak solution of (5.8) with $t$ fixed. This justifies that we call the unique $\mathrm{C}^{0}$-solution of $u^{\prime}(t)+\mathcal{A}_{W}^{\mathcal{H}} u(t)=0$ with $u(0)=u_{0} \in \overline{D\left(\mathcal{A}_{W}^{\mathcal{H}}\right)}$, or rather its first component, the (unique) solution of (5.8).

In the proof of Proposition 5.4 we have seen that $\left\{u \in W^{1, p}(\Omega) \cap \mathrm{C}(\bar{\Omega}): \mathcal{A} u \in\right.$ $\left.\mathrm{C}_{c}^{\infty}(\Omega)\right\}$ is dense in $\mathrm{C}(\bar{\Omega})$, which implies that $\mathcal{A}^{\mathcal{H}}$ and $\mathcal{A}^{\mathcal{C}}$ are densely defined. Now the same arguments as for Theorem 5.7 show that for every $u_{0} \in \mathrm{C}(\bar{\Omega})$ the unique $L^{2}$-solution of (5.8) is continuous on $[0, \infty) \times \bar{\Omega}$. 


\section{REFERENCES}

1. Robert A. Adams and John J. F. Fournier, Sobolev spaces, second ed., Pure and Applied Mathematics (Amsterdam), vol. 140, Elsevier/Academic Press, Amsterdam, 2003.

2. W. Arendt, G. Metafune, D. Pallara, and S. Romanelli, The Laplacian with Wentzell-Robin boundary conditions on spaces of continuous functions, Semigroup Forum 67 (2003), no. 2, 247-261.

3. Wolfgang Arendt, Resolvent positive operators and inhomogeneous boundary conditions, Ann. Scuola Norm. Sup. Pisa Cl. Sci. (4) 29 (2000), no. 3, 639-670.

4. Wolfgang Arendt and Michal Chovanec, Dirichlet regularity and degenerate diffusion, Trans. Amer. Math. Soc. 362 (2010), no. 11, 5861-5878.

5. Wolfgang Arendt and Reiner Schätzle, Semigroups generated by elliptic operators in non-divergence form on $C_{0}(\Omega)$, http://arxiv.org/abs/1010.1703v1, 2010.

6. R.F. Bass and P. Hsu, Some potential theory for reflecting Brownian motion in Hölder and Lipschitz domains, Ann. Probab. 19 (1991), no. 2, 486-508.

7. Philippe Bénilan, Michael G. Crandall, and Amnon Pazy, Nonlinear evolution equations in Banach spaces, Preprint book.

8. Daniel Daners and Pavel Drábek, A priori estimates for a class of quasi-linear elliptic equations, Trans. Amer. Math. Soc. 361 (2009), no. 12, 6475-6500.

9. Lawrence C. Evans and Ronald F. Gariepy, Measure theory and fine properties of functions, Studies in Advanced Mathematics, CRC Press, Boca Raton, FL, 1992.

10. M. Fukushima, A construction of reflecting barrier Brownian motions for bounded domains, Osaka J. Math. 4 (1967), 183-215.

11. David Gilbarg and Neil S. Trudinger, Elliptic partial differential equations of second order, Classics in Mathematics, Springer-Verlag, Berlin, 2001, Reprint of the 1998 edition.

12. J. A. Griepentrog, K. Gröger, H.-Chr. Kaiser, and J. Rehberg, Interpolation for function spaces related to mixed boundary value problems, Math. Nachr. 241 (2002), 110-120.

13. Jens A. Griepentrog and Lutz Recke, Linear elliptic boundary value problems with non-smooth data: normal solvability on Sobolev-Campanato spaces, Math. Nachr. 225 (2001), 39-74.

14. Jens André Griepentrog and Lutz Recke, Local existence, uniqueness and smooth dependence for nonsmooth quasilinear parabolic problems, J. Evol. Equ. 10 (2010), no. 2, 341-375.

15. P. Grisvard, Elliptic problems in nonsmooth domains, Monographs and Studies in Mathematics, vol. 24, Pitman (Advanced Publishing Program), Boston, MA, 1985 .

16. Konrad Gröger and Lutz Recke, Applications of differential calculus to quasilinear elliptic boundary value problems with non-smooth data, NoDEA Nonlinear Differential Equations Appl. 13 (2006), no. 3, 263-285.

17. Robert Haller-Dintelmann and Joachim Rehberg, Maximal parabolic regularity for divergence operators including mixed boundary conditions, J. Differential Equations 247 (2009), no. 5, 1354-1396.

18. Coercivity for elliptic operators and positivity of solutions on Lipschitz domains, Arch. Math. (Basel) 95 (2010), no. 5, 457-468.

19. Einar Hille and Ralph S. Phillips, Functional analysis and semi-groups, American Mathematical Society Colloquium Publications, vol. 31, American Mathematical Society, Providence, R. I., 1957, rev. ed. 
20. Petri Juutinen, Peter Lindqvist, and Juan J. Manfredi, On the equivalence of viscosity solutions and weak solutions for a quasi-linear equation, SIAM J. Math. Anal. 33 (2001), no. 3, 699-717 (electronic).

21. Hans-Christoph Kaiser, Hagen Neidhardt, and Joachim Rehberg, Classical solutions of quasilinear parabolic systems on two dimensional domains, NoDEA Nonlinear Differential Equations Appl. 13 (2006), no. 3, 287-310.

22. Gary M. Lieberman, The conormal derivative problem for elliptic equations of variational type, J. Differential Equations 49 (1983), no. 2, 218-257.

23. The natural generalization of the natural conditions of Ladyzhenskaya and Ural'tseva for elliptic equations, Comm. Partial Differential Equations 16 (1991), no. 2-3, 311-361.

24. Giuseppe Mingione, The Calderón-Zygmund theory for elliptic problems with measure data, Ann. Sc. Norm. Super. Pisa Cl. Sci. (5) 6 (2007), no. 2, 195-261.

25. R. Nittka, Regularity of solutions of linear second order elliptic and parabolic boundary value problems on Lipschitz domains, submitted; preprint at arXiv:0906.5285v1, 2009.

26. Elliptic and parabolic problems with Robin boundary conditions on Lipschitz domains, Ph.D. thesis, University of Ulm, March 2010.

27. James Serrin, Local behavior of solutions of quasi-linear equations, Acta Math. 111 (1964), 247-302.

28. R. E. Showalter, Monotone operators in Banach space and nonlinear partial differential equations, Mathematical Surveys and Monographs, vol. 49, American Mathematical Society, Providence, RI, 1997.

29. G.M. Troianiello, Elliptic differential equations and obstacle problems, The University Series in Mathematics, Plenum Press, New York, 1987.

30. Mahamadi Warma, The Robin and Wentzell-Robin Laplacians on Lipschitz domains, Semigroup Forum 73 (2006), no. 1, 10-30.

31. William P. Ziemer, Weakly differentiable functions, Graduate Texts in Mathematics, vol. 120, Springer-Verlag, New York, 1989.

Robin Nittka, University of Ulm, Institute of Applied Analysis, 89069 Ulm, GerMANY

E-mail address: robin.nittka@uni-ulm.de 